\title{
An Optimal Transmission Strategy for Kalman Filtering Over Packet Dropping Links With Imperfect Acknowledgements
}

\author{
Mojtaba Nourian, Member, IEEE, Alex S. Leong, Member, IEEE, Subhrakanti Dey, Senior Member, IEEE, \\ and Daniel E. Quevedo, Senior Member, IEEE
}

\begin{abstract}
This paper presents a novel design methodology for optimal transmission policies at a smart sensor to remotely estimate the state of a stable linear stochastic dynamical system. The sensor makes measurements of the process and forms estimates of the state using a local Kalman filter. The sensor transmits quantized information over a packet dropping link to the remote receiver. The receiver sends packet receipt acknowledgments back to the sensor via an erroneous feedback communication channel which is itself packet dropping. The key novelty of this formulation is that the smart sensor decides, at each discrete time instant, whether to transmit a quantized version of either its local state estimate or its local innovation. The objective is to design optimal transmission policies in order to minimize a long-term average cost function as a convex combination of the receiver's expected estimation error covariance and the energy needed to transmit the packets. Under high-resolution quantization assumptions, the optimal transmission policy is obtained by the use of dynamic programming techniques. Using the concept of submodularity, the optimality of a threshold policy in the case of scalar systems with perfect packet receipt acknowledgments is proved. Suboptimal solutions and their structural results are also discussed. Numerical results are presented, illustrating the performance of the optimal and suboptimal transmission policies.
\end{abstract}

Index Terms-High-resolution quantizer, Markov decision processes with imperfect state information, packet drops, state estimation, threshold policy, wireless sensor networks.

\section{INTRODUCTION}

$\mathbf{O}$ NE OF the important challenges in wireless-based networks is to improve system performance and reliability under resource (e.g., energy/power, computation, and communication) constraints. This concern is particularly crucial in industrial applications, such as remote sensing and real-time control, where a high level of reliability is usually required. As a consequence, it becomes of significant importance to investigate the impact of realistic wireless communication channel models in the area of state estimation and control of networked

Manuscript received August 8, 2013; revised August 9, 2013 and February 10, 2014; accepted May 6, 2014. Date of publication July 10, 2014; date of current version September 16, 2014. Recommended by Associate Editor K. H. Johansson.

M. Nourian and A. S. Leong are with the Department of Electrical and Electronic Engineering, The University of Melbourne, VIC 3010, Australia (e-mail: mojtaba.nourian@unimelb.edu.au; asleong@unimelb.edu.au).

S. Dey is with the Department of Engineering Sciences, Uppsala University, Uppsala SE-75 217, Sweden (e-mail: subhra.dey@signal.uu.se).

D. E. Quevedo is with the School of Electrical Engineering \& Computer Science, The University of Newcastle, Newcastle NSW 2308, Australia (e-mail: dquevedo@ieee.org).

Color versions of one or more of the figures in this paper are available online at http://ieeexplore.ieee.org.

Digital Object Identifier 10.1109/TCNS.2014.2337975 systems [1]. Two important limitations of wireless communication channels in these problem formulations include: 1) limited bandwidth and 2) information loss.

Among the many papers in the area of networked state estimation and control over bandwidth-limited channels, we first mention [2], which addresses the minimum data rate required for stability of a linear stochastic system with quantized measurements received through a finite rate channel. Recently, this work is extended to the general case of time-varying Markov digital communication channels in [3]. The reader is also referred to the survey [4] and the references therein.

Since the seminal work of [5], state estimation or Kalman filtering problems over packet dropping communication channels has been extensively studied (see, for example, [6]-[11], among others). The reader is also referred to the survey [12] and the references therein. In these problems, sensor measurements (or state estimates in the case of [6]) are grouped into packets which are transmitted over a packet dropping link. The focus in these works is on deriving conditions on the packet arrival rate in order to guarantee the stability of the Kalman filter. There are other works which are concerned with estimation performance (e.g., minimizing the expected estimation error covariance) rather than just stability. For instance, power allocation techniques have been applied to the Kalman filtering problem in [13]-[15] in order to improve the estimation performance and reliability.

Even though most of the works available in the literature focus on only one of the two mentioned communication limitations (limited bandwidth or information loss), some recent works attempt to address both limitations. In particular, the problem of minimum data rates for achieving bounded average state estimation error in linear systems over lossy channels is studied in [16] and [17] (see also [18]), while the problem of state control around a target state trajectory in the case of signal quantization and packet drops is investigated in [19] and [20]. The work in [21] concentrates on designing coding and decoding schemes to remotely estimate the state of a scalarstable stochastic linear system over a communication channel subject to quantization noise and packet loss.

Similar to [21], this paper is concerned with remote state estimation subject to quantization noise and packet drops. However, rather than considering fixed coding and decoding schemes, we are interested in designing optimal transmission policies at the smart sensor, choosing between sending the sensor's local state estimates or its local innovations. More 


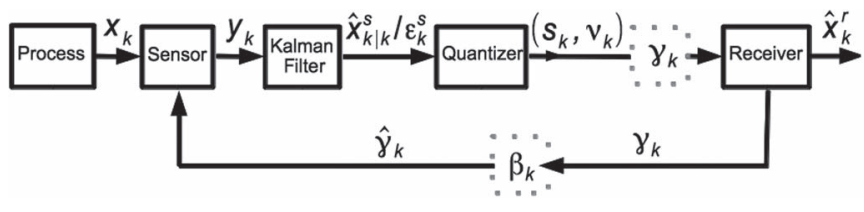

Fig. 1. System architecture.

specifically, we present a novel design methodology for optimal transmission policies at a smart sensor to remotely estimate the state of a stable (see Section VIII for some comments on extensions to unstable systems) linear stochastic dynamical system. The sensor makes measurements of the process and forms estimates of the state using a local Kalman filter (see Fig. 1). The sensor then transmits quantized (using a highresolution quantizer) information over a packet dropping link to the remote receiver. The sensor decides, at each time instant, whether to transmit a quantized version of either its local state estimate or its local innovation. The receiver runs a Kalman filter with random packet dropouts to minimize the estimation error covariances based on received measurements.

The packet reception probability is generally a function of the length of the packet, such that shorter packets (and, hence, lower required data rates) may result in higher packet receipt probabilities. Since the local innovation process has a smaller covariance, for a fixed packet reception probability, the quantized innovations require less energy to transmit than the quantized state estimates. However, due to the packet dropping link between the sensor and the remote estimator, if there has been a number of successive packet losses, then receiving a quantized state estimate might be more beneficial in reducing the estimation error covariance at the remote estimator than receiving the innovations. Thus, there is a tradeoff between whether the sensor should transmit its local state estimates or its local innovations. In general, knowledge at the sensor of whether its transmissions have been received is achieved via some feedback mechanism. Here, in addition to the case of the perfect packet receipt acknowledgments, we consider the more difficult problem where the feedback channel from the receiver to the sensor is an erroneous packet dropping link.

The objective is to design optimal transmission policies in order to minimize a long-term average (infinite-time horizon) cost function as a convex combination of the receiver's expected estimation error variance and the energy needed to transmit the packets. This problem is formulated as an average cost Markov decision process with imperfect state information. The optimal transmission policy is obtained by the use of dynamic programming techniques.

In summary, the main contributions of this paper are as follows.

1) Unlike a large number of papers focusing on only one of the two communication limitations (limited bandwidth or information loss), we consider both limitations, that is, remote state estimation subject to both quantization noise and packet drops.

2) Although recent works, such as [17] and [18], consider packet loss and data-rate constraints simultaneously, the focus of these papers is on stabilizability (implying only bounded estimation error) whereas the focus on our work is on the actual estimation error performance of the remote estimator (albeit for a stable system) and the optimization of a cost combining the long-term average of estimation error and transmission energy expenditure.

3) Unlike [21], which considers fixed coding and decoding schemes, we are interested in designing optimal transmission policies at the smart sensor, choosing between sending the sensor's local state estimates or its local innovations.

4) We consider the case of imperfect feedback acknowledgements, which is more difficult to analyze than the case of perfect feedback acknowledgements. We model the feedback channel by a general erasure channel with errors.

5) It is well known that the optimal solution obtained by a stationary control policy minimizing the infinite horizon control cost is computationally prohibitive. For the scalar case, we provide structural results on the optimal policy which lead to simple threshold policies which are optimal and yet very simple to implement.

6) Finally, also motivated by the computational burden for the optimal control solution in the general case of imperfect acknowledgments, we provide a suboptimal solution based on an estimate of the error covariance at the receiver. Numerical results are presented to illustrate the performance gaps between the optimal and suboptimal solutions.

The organization of this paper is as follows. The system model is given in Section II. The augmented state-space model at the remote receiver is constructed in Section III and the corresponding Kalman filtering equations are given. Section IV presents optimal transmission policy problems, together with their solutions, in both the cases of perfect and imperfect packet receipt acknowledgements. A suboptimal transmission scheme in the case of imperfect packet receipt acknowledgements is considered in Section V. For scalar systems, Section VI proves the optimality of the threshold transmission policy for the case of perfect packet receipt acknowledgements. Numerical simulations are given in Section VII.

We use the following notation. Let $(\Omega, \mathcal{F}, \mathbb{P})$ be a complete probability space. $\mathbb{E}$ denotes the expectation. Throughout this paper, the subscript or superscript $s$ are used for the sensor's quantities, and the superscript $r$ is used for the receiver's quantities. We say that a matrix $X>0$ if $X$ is positive definite, and $X \geq 0$ if $X$ is positive semi-definite.

\section{SyStem Model}

We start with a diagram of the system architecture that is shown in Fig. 1. Detailed descriptions of each part of the system are given below.

\section{A. Process Dynamics and Sensor Measurements}

We consider a stable uncontrolled linear time-invariant stochastic dynamical process

$$
x_{k+1}=A x_{k}+w_{k}, \quad k \geq 0
$$


where $x_{k} \in \mathbb{R}^{n}$ is the process state at instant $k \geq 0$, with $A$ being a Schur stable matrix, and $\left\{w_{k}: k \geq 0\right\}$ is a sequence of independent and identically distributed (i.i.d.) Gaussian noises with zero mean and covariance $\Sigma_{w} \geq 0$. The initial state of the process $x_{0}$ is a Gaussian random vector, independent of the process noise sequence $\left\{w_{k}: k \geq 0\right\}$, with mean $\bar{x}_{0}:=\mathbb{E}\left[x_{0}\right]$ and covariance $P_{x_{0}} \geq 0$.

The sensor measurements are obtained in the form

$$
y_{k}=C x_{k}+v_{k}, \quad k \geq 0
$$

where $y_{k} \in \mathbb{R}^{m}$ is the vector observation at instant $k \geq 0, C \in$ $\mathbb{R}^{m \times n}$, and $\left\{v_{k}: k \geq 0\right\}$ is a sequence of i.i.d. Gaussian noises, independent of both $x_{0}$ and $\left\{w_{k}: k \geq 0\right\}$, with zero mean and covariance $\Sigma_{v}>0$. We enunciate the following assumption:

(A1) We assume that $(A, C)$ is detectable.

\section{B. Local Kalman Filter at the Smart Sensor}

We assume that the sensor has some computational capabilities. In particular, it can run a local Kalman filter to reduce the effects of measurement noise, as in, for example, [6].

Denote the local sensor information at each instant $k$ by $\mathcal{Y}_{k}^{s}:=\sigma\left\{y_{t}: 0 \leq t \leq k\right\}$, which is the $\sigma$-field generated by the sensor measurements up to time $k$. We use the convention $\mathcal{Y}_{0}^{s}:=\{\emptyset, \Omega\}$. Then, the Kalman filtering and prediction estimates of the process state $x_{k}$ at the sensor are given by $\hat{x}_{k \mid k}^{s}=\mathbb{E}\left[x_{k} \mid \mathcal{Y}_{k}^{s}\right]$ and $\hat{x}_{k+1 \mid k}^{s}=\mathbb{E}\left[x_{k+1} \mid \mathcal{Y}_{k}^{s}\right]$, respectively.

We assume that the local Kalman filter has reached steady state. The stationary error-covariance is defined by $P_{s}=\lim _{k \rightarrow \infty} \mathbb{E}\left[\left(x_{k+1}-\hat{x}_{k+1 \mid k}^{s}\right)\left(x_{k+1}-\hat{x}_{k+1 \mid k}^{s}\right)^{T} \mid \mathcal{Y}_{k}^{s}\right]$, which is the solution of the algebraic Riccati equation (see, for example, [22])

$$
P_{s}=A P_{s} A^{T}+\Sigma_{w}-A P_{s} C^{T}\left(C P_{s} C^{T}+\Sigma_{v}\right)^{-1} C P_{s} A^{T} .
$$

The Kalman filter equations for $\hat{x}_{k \mid k}^{s}$ and $\hat{x}_{k+1 \mid k}^{s}$ are given by

$$
\begin{gathered}
\hat{x}_{k \mid k}^{s}=\hat{x}_{k \mid k-1}^{s}+K_{f}\left(y_{k}-C \hat{x}_{k \mid k-1}^{s}\right), \quad k \geq 0 \\
\hat{x}_{k+1 \mid k}^{s}=A \hat{x}_{k \mid k-1}^{s}+K_{s}\left(y_{k}-C \hat{x}_{k \mid k-1}^{s}\right), \quad k \geq 0
\end{gathered}
$$

with $\hat{x}_{0 \mid-1}^{s}:=\bar{x}_{0}$, where $K_{f}:=P_{s} C^{T}\left(C P_{s} C^{T}+\Sigma_{v}\right)^{-1}$ and $K_{s}:=A K_{f}$ are the stationary Kalman filtering and prediction gains, respectively. Denote the covariance of the local state estimate via $\Sigma_{s}:=\lim _{k \rightarrow \infty} \mathbb{E}\left[\left(\hat{x}_{k+1 \mid k}^{s}\right)\left(\hat{x}_{k+1 \mid k}^{s}\right)^{T} \mid \mathcal{Y}_{k}^{s}\right]$, which satisfies the stationary Lyapunov equation

$$
\Sigma_{s}=A \Sigma_{s} A^{T}+K_{s}\left(C P_{s} C^{T}+\Sigma_{v}\right) K_{s}^{T} .
$$

\section{Coding Alternatives at the Smart Sensor}

We define the innovation process ${ }^{1}$ at the sensor $\epsilon_{(\cdot)}$ as

$$
\epsilon_{k}^{s}=\hat{x}_{k \mid k}^{s}-\hat{x}_{k \mid k-1}^{s}=K_{f}\left(y_{k}-C \hat{x}_{k \mid k-1}^{s}\right), \quad k \geq 0 .
$$

\footnotetext{
${ }^{1}$ Note that $\epsilon_{k}^{s}$ is a linear transformed version of the true innovation process
} of Kalman filtering given by $\left(y_{k}-C \hat{x}_{k \mid k-1}^{s}\right)$.
As depicted in Fig. 1, the sensor communicates over a digital erasure channel with a remote receiver which utilizes the received data to calculate an estimate of the process state $x_{(\cdot)}$.

This work aims at investigating what data the smart wireless sensor should transmit to the receiver. Motivated by differential pulse-code modulation (PCM) techniques [23], [24], the digital sensor may convey either a vector quantized version of its local estimate or a vector quantized version of its innovation. Therefore, we may denote the packet sent by the sensor as

$$
s_{k}:=\left\{\begin{array}{ll}
\hat{x}_{k \mid k}^{s}+q_{k}^{x} & \text { if } \nu_{k}=1 \\
\epsilon_{k}^{s}+q_{k}^{\epsilon} & \text { if } \nu_{k}=0,
\end{array} \quad k \geq 0\right.
$$

where $\nu_{k} \in\{0,1\}$ is a decision variable which is transmitted to the receiver in addition to $s_{k}$. The sequence $\left\{\nu_{k}\right\}$ is designed at the sensor, see Section IV. In (8), $q_{(\cdot)}^{x}$ and $q_{(\cdot)}^{\epsilon}$ are the quantization noises resulting from encoding $\hat{x}_{k \mid k}^{s}$ and $\epsilon_{k}^{s}$, respectively. We note that in this paper the effects of the quantizer are only modelled via the additive quantization noise term in (8), which is assumed to be zero-mean white noise processes independent of the quantized signal. For high-rate quantization, such an approach is quite accurate (see Remark 2.1 below for the validity of this model to low-moderate rate quantization), since the quantization noises at high rates are approximately uncorrelated with the quantizer inputs [25], [26]. It is also reasonable to assume that the quantization noises, while uncorrelated to the inputs, have covariances which are proportional to the input covariances, i.e.,

$$
\begin{aligned}
& \Sigma_{q}^{x}:=\lim _{k \rightarrow \infty} \mathbb{E}\left[q_{k}^{x}\left(q_{k}^{x}\right)^{T}\right]=\alpha_{1} \lim _{k \rightarrow \infty} \mathbb{E}\left[\hat{x}_{k \mid k}^{s}\left(\hat{x}_{k \mid k}^{s}\right)^{T}\right] \\
& \Sigma_{q}^{\epsilon}:=\lim _{k \rightarrow \infty} \mathbb{E}\left[q_{k}^{\epsilon}\left(q_{k}^{\epsilon}\right)^{T}\right]=\alpha_{0} \lim _{k \rightarrow \infty} \mathbb{E}\left[\epsilon_{k}^{s}\left(\epsilon_{k}^{s}\right)^{T}\right]
\end{aligned}
$$

for given $\alpha_{0}, \alpha_{1} \geq 0$ which depend upon the quantizers and the bit rates used. ${ }^{2}$ We can obtain $\lim _{k \rightarrow \infty} \mathbb{E}\left[\hat{x}_{k \mid k}^{s}\left(\hat{x}_{k \mid k}^{s}\right)^{T}\right]=\Sigma_{s}+$ $K_{f}\left(C P_{s} C^{T}+\Sigma_{v}\right) K_{f}^{T}$ from (4), and $\lim _{k \rightarrow \infty} \mathbb{E}\left[\epsilon_{k}^{s}\left(\epsilon_{k}^{s}\right)^{T}\right]=$ $K_{f}\left(C P_{s} C^{T}+\Sigma_{v}\right) K_{f}^{T}$ from (7).

Consider a vector Gaussian source $s$ with $N=2^{n}$ quantizer levels where $n$ is the transmission rate (i.e., the number of bits transmitted per sample). Then, the quantization noise covariance of a high-resolution quantizer will be $\Sigma_{q} \approx \alpha \mathbb{E}\left[s s^{T}\right]$. For the case of asymptotically optimal lattice vector quantizers with Voronoi cell $S_{0}$, we have (see [28])

$$
\alpha=\frac{M\left(S_{0}\right) V^{\frac{2}{m}}}{\eta^{2}} \frac{\frac{2}{m} \ln N}{N^{\frac{2}{m}}}
$$

where $m$ represents the dimension of the vector to be quantized $\eta=\sqrt{1 / 2}, V=\left(\pi^{m / 2} / \Gamma(m / 2+1)\right)$

$$
M\left(S_{0}\right)=\frac{\frac{1}{k} \int_{S_{0}}\|x-y\|_{2}^{2} d x}{v\left(S_{0}\right)^{1+2 / m}}
$$

is the normalized moment of inertia of $S_{0}$, and $v\left(S_{0}\right)$ is the volume of $S_{0}$. For $m=1$, it can be shown that $\alpha$ reduces

\footnotetext{
${ }^{2}$ For an explanation on how the scaling factors $\alpha_{0}, \alpha_{1} \geq 0$ arise, see [27, p. 3860].
} 
to $\alpha=\left(4 \ln N / 3 N^{2}\right)$. For the case of "optimal" Lloyd-Max quantizers, we have $\alpha \sim\left(B_{m} / N^{2 / m}\right)$ (see [29]). However, the exact values of the constants $B_{m}$ are not known for dimensions $m \geq 3$. For $m=1$, we have $\alpha=\left(\pi \sqrt{3} / 2 N^{2}\right)$.

Remark 2.1: In principle, this additive white noise model for the quantization error is valid for high-resolution quantization. However, it has been reported by many works, including the seminal review paper by Gray and Neuhoff [30, p. 2358] that the high-resolution theory is fairly accurate for rates greater than or equal to $3 \mathrm{~b} /$ sample per signal dimension. More recent papers, such as [27], have reported similar results in designing decentralized linear estimation schemes with quantized innovations. Finally, the same quantization noise model has been used in a parallel work by Dey, Chiuso, and Schenato (see the extended online version of [21]). It has been shown in [21] that only 3 bits of quantization/sample for a convex combination of the (scalar) state estimate and the innovation signal at the transmitter achieve remote estimation error performance that is sufficiently close to the one predicted by the additive white noise model. Note that in the context of modern wireless local-area networks (LANs), communication rates on the order of megabits per second are quite common, implying that 3-5 bits of quantization per sample can be easily achieved. Thus, this approximation is a fairly accurate tool for analysis that is suitable for practical implementations as well.

Remark 2.2: Although quantization noise is generally modelled as uniformly distributed, it has been shown in a number of works that a Gaussian approximation to the quantization noise is valid at high-rate quantization. In particular, quantization noise due to lattice vector quantization (as used in this paper) approaches a white Gaussian noise in a divergence sense as the resolution increases [31].

Based on the aforementioned observations, we model the quantization noise processes $q_{k}^{x}$ and $q_{k}^{\epsilon}$ as zero-mean additive white Gaussian noise processes with covariances $\Sigma_{q}^{x}, \Sigma_{q}^{\epsilon}$, respectively. While this model is valid in principle at high rate quantization, it serves as a good approximation and a very useful analytical tool also at low-to-moderate rates of quantization.

In what follows, we allow the sensor to choose a varying rate of quantization in order to make the traces of the quantization noise covariances $\Sigma_{q}^{x}$ and $\Sigma_{q}^{\epsilon}$ the same. From (9), this implies that the data rates $n_{0}$ and $n_{1}$ for transmitting $\epsilon_{k}^{s}$ and $\hat{x}_{k \mid k}^{s}$ in the case of the lattice vector quantizer satisfy

$$
\begin{aligned}
\operatorname{Tr} \Sigma_{q}^{x} \equiv & \frac{M\left(S_{0}\right) V^{\frac{2}{m}}}{\eta^{2}} \frac{2 n_{1} \ln 2 / m}{2^{\frac{2 n_{1}}{m}}} \\
& \times \operatorname{Tr}\left(\Sigma_{s}+K_{f}\left(C P_{s} C^{T}+\Sigma_{v}\right) K_{f}^{T}\right)=\operatorname{Tr} \Sigma_{q}^{\epsilon} \\
\equiv & \frac{M\left(S_{0}\right) V^{\frac{2}{m}}}{\eta^{2}} \frac{2 n_{0} \ln 2 / m}{2^{\frac{2 n_{0}}{m}}} \operatorname{Tr}\left(K_{f}\left(C P_{s} C^{T}+\Sigma_{v}\right) K_{f}^{T}\right)
\end{aligned}
$$

and in the case of the Lloyd-Max quantizer

$$
\begin{aligned}
\operatorname{Tr} \Sigma_{q}^{x} & \equiv \frac{B_{m}}{2^{\frac{2 n_{1}}{m}}} \operatorname{Tr}\left(\Sigma_{s}+K_{f}\left(C P_{s} C^{T}+\Sigma_{v}\right) K_{f}^{T}\right) \\
& =\operatorname{Tr} \Sigma_{q}^{\epsilon} \equiv \frac{B_{m}}{2^{\frac{2 n_{0}}{m}}} \operatorname{Tr}\left(K_{f}\left(C P_{s} C^{T}+\Sigma_{v}\right) K_{f}^{T}\right) .
\end{aligned}
$$

If the resulting $n_{0}$ and $n_{1}$ are not integers, their nearest integers will be chosen as the transmission rates. Since $\Sigma_{s} \geq 0$, we have $n_{0} \leq n_{1}$ in the two cases above.

Since the local innovation process has a smaller stationary covariance and, hence, a smaller data rate to maintain a given packet receipt probability, transmitting $\epsilon_{k}^{s}$ should require less energy than transmitting $\hat{x}_{k \mid k}^{s}$ (see Section II-D). However, due to the packet dropping link between the sensor and the remote estimator, a number of successive packet losses imply that receiving $\hat{x}_{k \mid k}^{s}$ might be more beneficial in reducing the estimation error covariance at the remote estimator than receiving $\epsilon_{k}^{s}$. Thus, it is not immediately clear whether the sensor should transmit local estimates $\hat{x}_{k \mid k}^{s}$ or innovations $\epsilon_{k}^{s}$. This paper seeks to elucidate this dilemma in answering how to optimally design the control sequence $\left\{\nu_{k}: k \geq 0\right\}$ using causal information available at the sensor.

\section{Forward Erasure Communication Channel}

We assume that the forward communication channel between the sensor and the receiver is unreliable, see Fig. 1. This channel carries $\left\{\left(s_{k}, \nu_{k}\right): k \geq 0\right\}$ and is characterized by the transmission success process $\left\{\gamma_{k}: k \geq 0\right\}$, where $\gamma_{k}=1$ refers to successful reception of $\left(s_{k}, \nu_{k}\right)$, and $\gamma_{k}=0$ quantifies a dropout. Since the decision variable $\nu_{k}$ consists of only one bit of information, it can be easily sent along with $s_{k}$ as a header in the transmitted packet.

In this paper, we assume that $\gamma_{k}$ is a Bernoulli random variable with $\mathbb{P}\left(\gamma_{k}=1\right)=1-p$, where $p \in[0,1]$ is the packet loss probability. The packet loss probability is generally a function of the data rates, such that higher data rates result in higher packet loss probabilities. If $p_{b}$ is the error probability of sending one bit, then the packet loss probability of sending a packet of $n$ bits is given by

$$
p=1-\left(1-p_{b}\right)^{n}
$$

where the packet is assumed to be lost if an error occurs in any of its bits (e.g., when there is no channel coding used). We assume that the bit-error probability $p_{b}$ of a wireless communication channel depends on the transmission energy per bit $E_{b}$ such that $p_{b}$ decreases as $E_{b}$ increases. The bit-error probability $p_{b}$ can be computed for different combinations of channels and digital modulation schemes, for example, in the case of the additive white Gaussian noise (AWGN) channel with binary phase-shift keying (BPSK) modulation

$$
p_{b}=Q\left(\sqrt{\frac{2 E_{b}}{N_{0}}}\right)
$$

where $N_{0} / 2$ is the noise power spectral density, and $Q(x):=$ $(1 / \sqrt{2 \pi}) \int_{x}^{\infty} e^{-t^{2 / 2}} d t=(1 / 2) \operatorname{erfc}(x / \sqrt{2})$ is the $Q$-function [32]. As a consequence of (11) and (12), to obtain a fixed packet dropout probability, when innovations are sent, the transmit energy per bit will be lower than when local estimates are transmitted. In Section IV, we will further elucidate the situation and allocate power levels accordingly. 


\section{E. Erroneous Feedback Communication Channel}

In this paper, we will study the more realistic but complex case where acknowledgments are unreliable (see [33] and [34] for relevant models with an imperfect feedback mechanism). In this scenario, the packet loss process $\left\{\gamma_{k}, k \geq 0\right\}$ is not known to the sensor. Instead, the sensor receives an imperfect acknowledgment process $\left\{\hat{\gamma}_{k}, k \geq 0\right\}$ from the receiver. It is assumed that after the transmission of $y_{k}$ and before transmitting $y_{k+1}$, the sensor has access to the ternary process $\hat{\gamma}_{k} \in\{0,1,2\}$ where

$$
\hat{\gamma}_{k}= \begin{cases}0 \text { or } 1 & \text { if } \beta_{k}=1 \\ 2 & \text { if } \beta_{k}=0\end{cases}
$$

with given dropout probability $\eta \in[0,1]$ for the binary process $\left\{\beta_{k}: k \geq 0\right\}$, that is, $\mathbb{P}\left(\beta_{k}=0\right)=\eta$ for all $k \geq 0$. In the case $\beta_{k}=1$, a transmission error may occur, independent of all other random processes, with probability $\delta \in[0,1]$. We may model the erroneous feedback channel as a discrete memoryless erasure channel with errors depicted by a transition probability matrix

$$
\mathbb{A}=\left(a_{i j}\right)=\left[\begin{array}{ccc}
(1-\delta)(1-\eta) & \delta(1-\eta) & \eta \\
\delta(1-\eta) & (1-\delta)(1-\eta) & \eta
\end{array}\right]
$$

where $a_{i j}:=\mathbb{P}(\hat{\gamma}=j-1 \mid \gamma=i-1)$ for $i \in\{1,2\}$ and $j \in$ $\{1,2,3\}$. The present situation encompasses, as special cases, situations where no acknowledgments are available (UDPcase) and cases where acknowledgments are always available (TCP-case), see also [35] for a discussion in the context of closed-loop control with packet dropouts. The case of perfect packet receipt acknowledgments is a special case when $\eta$ and $\delta$ above are set to zero.

\section{AnAlysis of the System Model}

\section{A. Augmented State-Space Model at the Receiver}

To analyze the model considered in this paper, we write the dynamics of the augmented state $\theta_{k}:=\left[x_{k} \hat{x}_{k \mid k-1}^{s}\right]^{T}$ which we want to estimate at the remote receiver as

$$
\theta_{k+1}=\mathcal{A} \theta_{k}+\xi_{k}
$$

where $\mathcal{A}:=\left[\begin{array}{llll}A & 0 & K_{s} C A-K_{s} C\end{array}\right]$, and $\xi_{k}:=\left[\begin{array}{lll}w_{k} & K_{s} & v_{k}\end{array}\right]$ by (1), (2), and (5). From (8), the observation is given by $z_{k}=$ $\nu_{k}\left(\hat{x}_{k \mid k}^{s}+q_{k}^{x}\right)+\left(1-\nu_{k}\right)\left(\epsilon_{k}^{s}+q_{k}^{\epsilon}\right)$ or, equivalently,

$$
z_{k}=\mathcal{C}\left(\nu_{k}\right) \theta_{k}+\zeta_{k}
$$

where $C\left(\nu_{k}\right):=\left[K_{f} C \nu_{k} I-K_{f} C\right], \quad$ and $\quad \zeta_{k}:=K_{f} v_{k}+$ $v_{k} q_{k}^{x}+\left(1-v_{k}\right) q_{k}^{\epsilon}$ by (2), (4), and (7) (note that $K_{f} C$ is a square matrix). We note that $\left\{\xi_{k}: k \geq 0\right\}$ and $\left\{\zeta_{k}: k \geq 0\right\}$ are zero-mean noise processes. The covariance of the process $\left\{\xi_{k}: k \geq 0\right\}$ is

$$
Q:=E\left[\xi_{k} \xi_{k}^{T}\right]=\left[\begin{array}{cc}
\Sigma_{w} & 0 \\
0 & K_{s} \Sigma_{v} K_{s}^{T}
\end{array}\right] \geq 0
$$

while the covariance of the process $\left\{\zeta_{k}: k \geq 0\right\}$ is given by

$$
R\left(\nu_{k}\right):=E\left[\zeta_{k} \zeta_{k}^{T}\right]=K_{f} \Sigma_{v} K_{f}^{T}+\nu_{k}^{2} \Sigma_{q}^{x}+\left(1-\nu_{k}\right)^{2} \Sigma_{q}^{\epsilon} \geq 0 .
$$

The matrix $S$, which models the correlation between the augmented state process noise $\left\{\xi_{k}: k \geq 0\right\}$ and the measurement noise $\left\{\zeta_{k}: k \geq 0\right\}$, is given by

$$
S:=E\left[\xi_{k} \zeta_{k}^{T}\right]=\left[\begin{array}{c}
0 \\
K_{s} \Sigma_{v} K_{f}^{T}
\end{array}\right] .
$$

\section{B. Kalman Filter at the Receiver}

We assume that the receiver knows whether dropouts occurred or not. At instances where sensor packets are received, the decision variable $\nu_{k}$ is also known. Therefore, the information at the receiver at time $k, \mathcal{Y}_{k}^{r}$, is given by the $\sigma$-field $\sigma\left\{\gamma_{t}, \gamma_{t} \nu_{t}, \gamma_{t} z_{t}: 0 \leq t \leq k\right\}$. We use the convention $\mathcal{Y}_{0}^{r}:=$ $\{\emptyset, \Omega\}$. At any instant $k$, the receiver estimates the process state $x_{k}$ through estimation of the augmented state $\theta_{k}$ based on the information $\mathcal{Y}_{k-1}^{r}$. We denote the conditional expectation and the associated estimation error covariance of the augmented state $^{3}$ as $\hat{\theta}_{k}:=\mathbb{E}\left[\theta_{k} \mid \mathcal{Y}_{k-1}^{r}\right]$ and

$$
\mathbf{P}_{k}:=\mathbb{E}\left[\left(\theta_{k}-\hat{\theta}_{k}\right)\left(\theta_{k}-\hat{\theta}_{k}\right)^{T} \mid \mathcal{Y}_{k-1}^{r}\right]=\left[\begin{array}{cc}
P_{k}^{1,1} & P_{k}^{1,2} \\
P_{k}^{1,2} & P_{k}^{2,2}
\end{array}\right] .
$$

Let $\hat{x}_{k}^{r}:=\mathbb{E}\left[x_{k} \mid \mathcal{Y}_{k-1}^{r}\right]$. Then

$$
P_{k}^{1,1} \equiv \mathbb{E}\left[\left(x_{k}-\hat{x}_{k}^{r}\right)\left(x_{k}-\hat{x}_{k}^{r}\right)^{T} \mid \mathcal{Y}_{k-1}^{r}\right]
$$

is the state estimation error covariance at the receiver at time $k$. The estimation error covariance $\mathbf{P}_{(\cdot)}$ satisfies the following random Riccati equation of Kalman filtering with correlated process and measurement noises:

$$
\begin{aligned}
& \mathbf{P}_{k+1}=\mathcal{A} \mathbf{P}_{k} \mathcal{A}^{T}+Q-\gamma_{k}\left[\mathcal{A} \mathbf{P}_{k} \mathcal{C}^{T}\left(\nu_{k}\right)+S\right] \\
& \times\left[\mathcal{C}\left(\nu_{k}\right) \mathbf{P}_{k} \mathcal{C}^{T}\left(\nu_{k}\right)+R\left(\nu_{k}\right)\right]^{-1}\left[\mathcal{A} \mathbf{P}_{k} \mathcal{C}^{T}\left(\nu_{k}\right)+S\right]^{T} .
\end{aligned}
$$

Note that $\gamma_{k}$ appears as a random coefficient in the Riccati (15).

Theorem 3.1: The estimation error covariance $\mathbf{P}_{(\cdot)}$ of the augmented system is of the form

$$
\mathbf{P}_{k}=\left[\begin{array}{cc}
P_{k}^{1,1} & P_{k}^{1,1}-P_{s} \\
P_{k}^{1,1}-P_{s} & P_{k}^{1,1}-P_{s}
\end{array}\right], \quad k \geq 0 .
$$

Proof: See Appendix A.

Theorem 3.1 is useful for obtaining numerical solutions of the stochastic control problems considered in the next section. It reduces the size of the state space which we need to consider.

\section{Optimal Transmission Policy Problem}

Based on the discussion in Section II-B, the decision of whether to send the innovation $\epsilon_{k}^{s}$, that is, set $\nu_{k}=0$, or the state estimate $\hat{x}_{k \mid k}^{s}$, that is, set $\nu_{k}=1$, will result in bit rates $n_{0} \equiv n\left(\nu_{k}=0\right)$ or $n_{1} \equiv n\left(\nu_{k}=1\right)$, respectively, where $n_{0} \leq n_{1}$.

\footnotetext{
${ }^{3}$ Note that if the quantization noise distribution departs from the assumed Gaussianity (Remark 2.2), then the filter at the receiver should be interpreted as the best linear filter. The quantities $\hat{\theta}_{k}, \mathbf{P}_{k}$ will represent the corresponding estimate and its covariance, and will only be an approximation for the conditional mean and error covariance.
} 
To maintain a fixed packet loss probability $p$, these bit rates yield different bit-error probabilities $p_{b}^{0}$ and $p_{b}^{1}$ where

$$
p_{b}^{0}=1-(1-p)^{\frac{1}{n_{0}}} \geq p_{b}^{1}=1-(1-p)^{\frac{1}{n_{1}}}
$$

by (11) and the fact that $n_{0} \leq n_{1}$. The required transmission energies for bit-error probabilities $p_{b}^{0}$ and $p_{b}^{1}$ will be denoted by $E_{b}^{0}$ and $E_{b}^{1}$, respectively. Since the transmission energy is a decreasing function of the bit-error probability, we have $E_{b}^{0} \leq$ $E_{b}^{1}$. For example, in the case of AWGN channel with BPSK modulation, (12) implies that

$$
E_{b}^{0}=N_{0} \times\left(\operatorname{erfc}^{-1}\left(2 p_{b}^{0}\right)\right)^{2}, \quad E_{b}^{1}=N_{0} \times\left(\operatorname{erfc}^{-1}\left(2 p_{b}^{1}\right)\right)^{2}
$$

where $\operatorname{erfc}^{-1}($.$) is the inverse complementary error function,$ which is monotonically decreasing.

We define the energy per packet of $n$ bits at time $k$ as $J\left(\nu_{k}\right)=n_{\nu_{k}} \times E_{b}^{\nu_{k}}$ which depends on the control variable $\nu_{k} \in\{0,1\}$.

We now aim to design optimal transmission policies in order to minimize a convex combination of the trace of the receiver's expected estimation error variance and the amount of energy required at the sensor for sending the packet to the receiver. This optimization problem is formulated as a long term average (infinite-time horizon) stochastic control problem

$$
\begin{aligned}
& \min _{\left\{\nu_{k}\right\}} \limsup _{T \rightarrow \infty} \frac{1}{T} \sum_{k=0}^{T-1} \mathbb{E}\left[\lambda \operatorname{Tr} P_{k+1}^{1,1}+\right.(1-\lambda) J\left(\nu_{k}\right) \mid \\
&\left.\left\{\hat{\gamma}_{l}\right\}_{0}^{k-1},\left\{\nu_{l}\right\}_{0}^{k}, P_{x_{0}}\right]
\end{aligned}
$$

where $\lambda \in[0,1]$ is the weight, and $P_{k+1}^{1,1}$ is the submatrix of $\mathbf{P}_{k+1}$ [see (14)] obtained from the Riccati (15). To take into account the fact that acknowledgements are unreliable, the expectation in (17) is conditioned on the transmission success process of the feedback channel $\left\{\hat{\gamma}_{l}\right\}$ instead of the packet loss acknowledgment process of the forward channel $\left\{\gamma_{l}\right\}_{l=0}$. Thus, in problem (17), $\nu_{k}$ can only depend on $\left\{\hat{\gamma}_{l}\right\}_{0}^{k-1},\left\{\nu_{l}\right\}_{0}^{k}$, and $P_{x_{0}}$. Therefore, this formulation falls within the general framework of stochastic control problems with imperfect state information.

\section{A. Case of Perfect Packet Receipt Acknowledgments}

First, let us assume that the smart sensor has perfect information about whether the packets have been received at the remote estimator or not, that is, $\eta$ and $\delta$ are set to zero in Section II-E. The optimization problem (17) is then reduced to a stochastic control problem with perfect state information

$$
\begin{aligned}
\min _{\left\{\nu_{k}\right\}} \limsup _{T \rightarrow \infty} \frac{1}{T} \sum_{k=0}^{T-1} \mathbb{E}\left[\lambda \operatorname{Tr} P_{k+1}^{1,1}+(1-\lambda) J\left(\nu_{k}\right) \mid\right. \\
\left.\left\{\gamma_{l}\right\}_{0}^{k-1},\left\{\nu_{l}\right\}_{0}^{k}, P_{x_{0}}\right]
\end{aligned}
$$

which may be written as

$$
\min _{\left\{\nu_{k}\right\}} \limsup _{T \rightarrow \infty} \frac{1}{T} \sum_{k=0}^{T-1} \mathbb{E}\left[\lambda \operatorname{Tr} P_{k+1}^{1,1}+(1-\lambda) J\left(\nu_{k}\right) \mid \mathbf{P}_{k}, \nu_{k}\right]
$$

due to the fact that $\mathbf{P}_{k}$ is a deterministic function of $\left\{\gamma_{l}\right\}_{l=0}^{k-1}$, $\left\{\nu_{l}\right\}_{l=0}^{k-1}$, and $P_{x_{0}}$. Denote

$$
\begin{aligned}
\mathcal{L}(\mathbf{P}, \gamma, \nu):= & \mathcal{A} \mathbf{P} \mathcal{A}^{T}+Q-\gamma\left[\mathcal{A} \mathbf{P} \mathcal{C}^{T}(\nu)+S\right] \\
& \times\left[\mathcal{C}(\nu) \mathbf{P} \mathcal{C}^{T}(\nu)+R(\nu)\right]^{-1}\left[\mathcal{A} \mathbf{P} \mathcal{C}^{T}(\nu)+S\right]^{T} \\
\equiv & {\left[\begin{array}{cc}
\mathcal{L}^{1,1}(\mathbf{P}, \gamma, \nu) & \mathcal{L}^{1,1}(\mathbf{P}, \gamma, \nu)-P_{s} \\
\mathcal{L}^{1,1}(\mathbf{P}, \gamma, \nu)-P_{s} & \mathcal{L}^{1,1}(\mathbf{P}, \gamma, \nu)-P_{s}
\end{array}\right] }
\end{aligned}
$$

as the random Riccati equation operator (see Theorem 3.1), where matrices $\mathcal{A}, Q, \mathcal{C}, S$, and $R$ are given in Section III-A.

Theorem 4.1 (Perfect Packet Receipt Acknowledgments): Independent of the initial estimation error variance $P_{x_{0}}$, the value of problem (18) is given by $\rho$, which is the solution of the average cost optimality (Bellman) equation

$$
\begin{array}{r}
\rho+V(\mathbf{P})=\min _{\nu \in\{0,1\}}\left(\mathbb{E}\left[\lambda \operatorname{Tr} \mathcal{L}^{1,1}(\mathbf{P}, \gamma, \nu)+(1-\lambda) J(\nu) \mid \mathbf{P}, \nu\right]\right. \\
+\mathbb{E}[V(\mathcal{L}(\mathbf{P}, \gamma, \nu)) \mid \mathbf{P}, \nu])
\end{array}
$$

where $V$ is called the relative value function.

Proof: The proof follows from the dynamic programming principle for average cost stochastic control problems (see, e.g., [36, Prop. 7.4.1]).

The stationary solution to the problem (18) is then given by

$$
\begin{aligned}
\nu^{o}(\mathbf{P})= & \arg \min _{\nu \in\{0,1\}}\left(\mathbb { E } \left[\lambda \operatorname{Tr} \mathcal{L}^{1,1}(\mathbf{P}, \gamma, \nu)\right.\right. \\
& +(1-\lambda) J(\nu) \mid \mathbf{P}, \nu] \mathbb{E}[V(\mathcal{L}(\mathbf{P}, \gamma, \nu)) \mid \mathbf{P}, \nu])
\end{aligned}
$$

where $V(\cdot)$ is the solution to (20).

Remark 4.1: Equation (20), together with the control policy $\nu^{o}$ defined in (21), is known as the average cost optimality equations. If a control $\nu^{o}$, a measurable function $V$, and a constant $\rho$ exist which solve (20) and (21), then the strategy $\nu^{o}$ is optimal, and $\rho$ is the optimal cost in the sense that

$$
\limsup _{T \rightarrow \infty} \frac{1}{T} \sum_{k=0}^{T-1} \mathbb{E}\left[\lambda \operatorname{Tr} P_{k+1}^{1,1}+(1-\lambda) J\left(\nu_{k}\right) \mid \nu_{k}=\nu^{o}\left(\mathbf{P}_{k}\right)\right]=\rho
$$

and for any other control policy $\left\{\nu_{k} \in\{0.1\}: k \geq 0\right\}$

$$
\limsup _{T \rightarrow \infty} \frac{1}{T} \sum_{k=0}^{T-1} \mathbb{E}\left[\lambda \operatorname{Tr} P_{k+1}^{1,1}+(1-\lambda) J\left(\nu_{k}\right) \mid \nu_{k}\right] \geq \rho .
$$

The reader is referred to [37] for a proof of the average cost optimality equations and related results. We solve the Bellman equation with the use of the relative value iteration algorithm (see [36, Ch. 7]).

In (20), the term $\mathbb{E}\left[\mathcal{L}^{1,1}(\mathbf{P}, \gamma, \nu) \mid \mathbf{P}, \nu\right]$ is the submatrix [similar to (14)] of the following matrix:

$$
\begin{gathered}
\mathbb{E}[\mathcal{L}(\mathbf{P}, \gamma, \nu) \mid \mathbf{P}, \nu]=A \mathbf{P} A^{T}+Q-(1-p) \times\left[A \mathbf{P} C^{T}(\nu)+S\right] \\
\times\left[C(\nu) \mathbf{P} C^{T}(\nu)+R(\nu)\right]^{-1}\left[A \mathbf{P} C^{T}(\nu)+S\right]^{T}
\end{gathered}
$$

where $p$ is the packet loss probability of the forward erasure communication channel given in Section II-D. 


\section{B. Case of Imperfect Packet Receipt Acknowledgments}

In a more practical formulation of problem (17), the smart sensor does not have perfect knowledge about whether its transmissions have been received at the receiver. Hence, at time $k$, the sensor has only "imperfect state information" about $\left\{\mathbf{P}_{t}: 1 \leq t \leq k\right\}$ via the acknowledgment process $\left\{\hat{\gamma}_{t}, 0 \leq\right.$ $t \leq k-1\}$. We will reduce the optimization problem (17) to a stochastic control problem with perfect state information by using the notion of information-state [38]. For $k \geq 0$, denote $z^{k}:=\left\{\hat{\gamma}_{0}, \cdots, \hat{\gamma}_{k}, \nu_{0}, \cdots, \nu_{k-1}, P_{x_{0}}\right\}$ as all observations about the receiver's Kalman filtering state estimation error covariance at the sensor after transmission at time $k$ and before transmission at time $k+1$. We set $z^{-1}:=\left\{P_{x_{0}}\right\}$. The information-state is defined by

$$
f_{k+1}\left(\mathbf{P}_{k+1} \mid z^{k}, \nu_{k}\right)=\mathbb{P}\left(\mathbf{P}_{k+1} \mid z^{k}, \nu_{k}\right), \quad k \geq 0
$$

which is the conditional probability of the estimation error covariance $\mathbf{P}_{k+1}$ given $\left(z^{k}, \nu_{k}\right)$. The following lemma shows how $f_{k+1}\left(\cdot \mid z^{k}, \nu_{k}\right)$ can be determined from $f_{k}\left(\cdot \mid z^{k-1}, \nu_{k-1}\right)$ together with $\hat{\gamma}_{k}$ and $\nu_{k}$.

Lemma 4.1: The information-state $f_{(\cdot)}$ satisfies the recursion

$$
\begin{aligned}
& f_{k+1}\left(\mathbf{P}_{k+1} \mid z^{k}, \nu_{k}\right) \\
& =\sum_{\gamma_{k} \in\{0,1\}}\left[\int_{\mathbf{P}_{k}}\left(\mathbb{P}\left(\mathbf{P}_{k+1} \mid \mathbf{P}_{k}, \gamma_{k}, \nu_{k}\right) \times f_{k}\left(\mathbf{P}_{k} \mid z^{k-1}, \nu_{k-1}\right)\right) d \mathbf{P}_{k}\right. \\
& \left.\quad \times \frac{\mathbb{P}\left(\hat{\gamma}_{k} \mid \gamma_{k}\right) \times \mathbb{P}\left(\gamma_{k}\right)}{\sum_{\gamma_{k} \in\{0,1\}} \mathbb{P}\left(\hat{\gamma}_{k} \mid \gamma_{k}\right) \times \mathbb{P}\left(\gamma_{k}\right)}\right] \\
& =: \Phi\left[f_{k}\left(\cdot \mid z^{k-1}, \nu_{k-1}\right), \hat{\gamma}_{k}, \nu_{k}\right]\left(\mathbf{P}_{k+1}\right) . \quad k \geq 0
\end{aligned}
$$

with $f_{0}\left(\mathbf{P}_{0} \mid z^{-1}\right)=\delta\left(\mathbf{P}_{0}\right)$, where $\delta$ is the Dirac delta function.

Proof: See Appendix B.

Note that in (24), the probabilities $\mathbb{P}\left(\hat{\gamma}_{k} \mid \gamma_{k}\right)$ can be obtained from the probability transition matrix $\mathbb{A}$ in (13). It is important to note that $\Phi$ in (24) depends on the entire function $f_{k}\left(\cdot \mid z^{k-1}, \nu_{k-1}\right)$ and not just on its value at any particular $\mathbf{P}_{k}$.

We now reduce problem (17) to a problem with perfect state information, where its state is given by the information state $f_{(.)}$, which evolves based on the recursion (24). Define the class of matrices $\mathbb{S}$ as

$$
\mathbb{S}:=\left\{\mathbf{P}=\left[\begin{array}{cc}
P & P-P_{s} \\
P-P_{s} & P-P_{s}
\end{array}\right]: P \geq P_{s}\right\}
$$

Theorem 4.2 (Imperfect Packet Receipt Acknowledgments): Independent of the initial estimation error variance $P_{x_{0}}$, the value of problem (17) is given by $\rho$, which is the solution of the average cost optimality (Bellman) equation

$$
\begin{array}{r}
\rho+V(\pi)=\min _{\nu \in\{0,1\}}\left(\mathbb{E}\left[\lambda \operatorname{Tr} \mathcal{L}^{1,1}(\mathbf{P}, \gamma, \nu)+(1-\lambda) J(\nu) \mid \pi, \nu\right]\right. \\
+\mathbb{E}[V(\Phi(\pi, \hat{\gamma}, \nu)) \mid \pi, \nu])
\end{array}
$$

for $\pi \in \Pi$, where the operator $\Phi$ is defined in (24), $V$ is the relative value function, and $\Pi$ is the space of probability density functions on matrices $\mathbb{S}$ of the form (25).

Proof: The proof follows from the dynamic programming principle for stochastic control problems with imperfect state information (see [38, Theor. 7.1]).
Note that in (26), the state is the entire probability density function $\pi$ which takes its values in the space of probability densities $\Pi$. We may write the terms in (26) as

$$
\begin{aligned}
& \mathbb{E}[\mathcal{L}(\mathbf{P}, \gamma, \nu) \mid \pi, \nu] \\
& =\int_{\mathbf{P}}\left(\mathcal{A} \mathbf{P} \mathcal{A}^{T}+Q\right) \pi(\mathbf{P}) d \mathbf{P} \\
& -(1-p) \times \int_{\mathbf{P}}\left(\left[\mathcal{A} \mathbf{P} \mathcal{C}^{T}(\nu)+S\right]\left[\mathcal{C}(\nu) \mathbf{P} \mathcal{C}^{T}(\nu)+R(\nu)\right]^{-1}\right. \\
& \left.\quad \times\left[\mathcal{A} \mathbf{P} \mathcal{C}^{T}(\nu)+S\right]^{T}\right) \pi(\mathbf{P}) d \mathbf{P}
\end{aligned}
$$

and $\mathbb{E}[V(\Phi(\pi, \hat{\gamma}, \nu)) \mid \pi, \nu]=\mathbb{P}(\hat{\gamma}=0) V(\Phi(\pi, 0, \nu))+\mathbb{P}(\hat{\gamma}=$ 1) $V(\Phi(\pi, 1, \nu))+\mathbb{P}(\hat{\gamma}=2) V(\Phi(\pi, 2, \nu))$.

\section{Suboptimal Transmission Policy Problem}

To obtain the optimal transmission strategy in the case of imperfect packet receipt acknowledgments presented in Section IV-B, we need to compute the solution of the Bellman equation (26) in the space of probability density functions $\Pi$, which is computationally demanding. In this section, we consider suboptimal policies which are computationally much less intense than finding the optimal solution.

We formulate the suboptimal optimization problem as

$$
\min _{\left\{\nu_{k}\right\}} \limsup _{T \rightarrow \infty} \frac{1}{T} \sum_{k=0}^{T-1} \mathbb{E}\left[\lambda \operatorname{Tr} \hat{P}_{k+1}^{1,1}+(1-\lambda) J\left(\nu_{k}\right) \mid \hat{\mathbf{P}}_{k}, \nu_{k}\right]
$$

where $\hat{P}_{(\cdot)}^{1,1}$ is the submatrix [similar to (14)] of $\hat{\mathbf{P}}_{(\cdot)}$ which is an estimate of $\mathbf{P}_{(\cdot)}$ computed by the sensor based on the following recursive equations (with $\hat{\mathbf{P}}_{0}=\mathbf{P}_{0}$ ):

i) In the case $\hat{\gamma}_{k}=0$, we have

$$
\begin{aligned}
\hat{\mathbf{P}}_{k+1}:= & \left(\mathcal{A} \hat{\mathbf{P}}_{k} \mathcal{A}^{T}+Q\right) \times \frac{\mathbb{P}\left(\hat{\gamma}_{k}=0 \mid \gamma_{k}=0\right) \times \mathbb{P}\left(\gamma_{k}=0\right)}{\sum_{\gamma_{k} \in\{0,1\}} \mathbb{P}\left(\hat{\gamma}_{k}=0 \mid \gamma_{k}\right) \times \mathbb{P}\left(\gamma_{k}\right)} \\
+ & \left(\mathcal{A} \hat{\mathbf{P}}_{k} \mathcal{A}^{T}+Q-\left[\mathcal{A} \hat{\mathbf{P}}_{k} \mathcal{C}^{T}\left(\nu_{k}\right)+S\right]\right. \\
& \times\left[\mathcal{C}\left(\nu_{k}\right) \hat{\mathbf{P}}_{k} C^{T}\left(\nu_{k}\right)+R\left(\nu_{k}\right)\right]^{-1} \\
& \left.\times\left[\mathcal{A} \hat{\mathbf{P}}_{k} \mathcal{C}^{T}\left(\nu_{k}\right)+S\right]^{T}\right) \\
& \times \frac{\mathbb{P}\left(\hat{\gamma}_{k}=0 \mid \gamma_{k}=1\right) \times \mathbb{P}\left(\gamma_{k}=1\right)}{\sum_{\gamma_{k} \in\{0,1\}} \mathbb{P}\left(\hat{\gamma}_{k}=0 \mid \gamma_{k}\right) \times \mathbb{P}\left(\gamma_{k}\right)}
\end{aligned}
$$

ii) In the case $\hat{\gamma}_{k}=1$, we have

$$
\begin{aligned}
\hat{P}_{k+1}:= & \left(\mathcal{A} \hat{\mathbf{P}}_{k} \mathcal{A}^{T}+Q\right) \times \frac{\mathbb{P}\left(\hat{\gamma}_{k}=1 \mid \gamma_{k}=0\right) \times \mathbb{P}\left(\gamma_{k}=0\right)}{\sum_{\gamma_{k} \in\{0,1\}} \mathbb{P}\left(\hat{\gamma}_{k}=1 \mid \gamma_{k}\right) \times \mathbb{P}\left(\gamma_{k}\right)} \\
+ & \left(\mathcal{A} \hat{\mathbf{P}}_{k} \mathcal{A}^{T}+Q-\left[\mathcal{A} \hat{\mathbf{P}}_{k} \mathcal{C}^{T}\left(\nu_{k}\right)+S\right]\right. \\
& \times\left[\mathcal{C}\left(\nu_{k}\right) \hat{\mathbf{P}}_{k} C^{T}\left(\nu_{k}\right)+R\left(\nu_{k}\right)\right]^{-1} \\
& \left.\times\left[\mathcal{A} \hat{\mathbf{P}}_{k} \mathcal{C}^{T}\left(\nu_{k}\right)+S\right]^{T}\right) \\
& \times \frac{\mathbb{P}\left(\hat{\gamma}_{k}=1 \mid \gamma_{k}=1\right) \times \mathbb{P}\left(\gamma_{k}=1\right)}{\sum_{\gamma_{k} \in\{0,1\}} \mathbb{P}\left(\hat{\gamma}_{k}=1 \mid \gamma_{k}\right) \times \mathbb{P}\left(\gamma_{k}\right)}
\end{aligned}
$$


iii) In the case $\hat{\gamma}_{k}=2$, we have

$$
\begin{aligned}
\hat{P}_{k+1}: & =\mathcal{A} \hat{\mathbf{P}}_{k} \mathcal{A}^{T}+Q-\mathbb{P}\left(\gamma_{k}=1\right) \times\left[\mathcal{A} \hat{\mathbf{P}}_{k} \mathcal{C}^{T}\left(\nu_{k}\right)+S\right] \\
& \times\left[\mathcal{C}\left(\nu_{k}\right) \hat{\mathbf{P}}_{k} \mathcal{C}^{T}\left(\nu_{k}\right)+R\left(\nu_{k}\right)\right]^{-1}\left[\mathcal{A} \hat{\mathbf{P}}_{k} \mathcal{C}^{T}\left(\nu_{k}\right)+S\right]^{T} .
\end{aligned}
$$

The reason why the solution to the stochastic control problem (27) is only suboptimal is that the true error covariance matrix $\mathbf{P}_{(\cdot)}$ in (17) is replaced by its estimate $\hat{\mathbf{P}}_{(\cdot)}$ in (27). The intuition behind these recursive equations can be explained as follows. Note that in the case of perfect feedback acknowledgements (15), the error covariance is updated as $\mathbf{P}_{k+1}=\mathcal{A} \mathbf{P}_{k} \mathcal{A}^{T}+Q$ in case $\gamma_{k}=0$, and $\mathbf{P}_{k+1}=\mathcal{A} \mathbf{P}_{k} \mathcal{A}^{T}+Q-\gamma_{k}\left[\mathcal{A} \mathbf{P}_{k} \mathcal{C}^{T}\left(\nu_{k}\right)+S\right] \times$ $\left[\mathcal{C}\left(\nu_{k}\right) \mathbf{P}_{k} \mathcal{C}^{T}\left(\nu_{k}\right)+R\left(\nu_{k}\right)\right]^{-1} \times\left[\mathcal{A} \mathbf{P}_{k} \mathcal{C}^{T}\left(\nu_{k}\right)+S\right]^{T}$ in case $\gamma_{k}=1$. In our imperfect acknowledgement model, even when $\beta_{k}=1$, errors can occur such that $\hat{\gamma}_{k}=0$ is received when $\gamma_{k}=1$, and $\hat{\gamma}_{k}=1$ is received when $\gamma_{k}=0$. Thus, the recursions given in i) and ii) are the weighted combinations of the error covariance recursions (based on the Bayes' rule using corresponding error event probabilities) in the case of perfect feedback acknowledgements. In the case $\hat{\gamma}_{k}=2$ where an erasure occurs, taking the average of the error covariances in the cases $\gamma_{k}=0$ and $\gamma_{k}=1$ is intuitively a reasonable thing to do, which motivates the recursion in iii).

Note that $\mathbb{P}\left(\hat{\gamma}_{k}\right)=\sum_{\gamma_{k} \in\{0,1\}} \mathbb{P}\left(\hat{\gamma}_{k} \mid \gamma_{k}\right) \mathbb{P}\left(\gamma_{k}\right)$, where the conditional probabilities are given in Section II-E. This, together with the recursive equations of $\hat{\mathbf{P}}_{(\cdot)}$, implies that the expression $\mathbb{E}\left[\hat{P}_{k+1}^{1,1} \mid \hat{\mathbf{P}}_{k}, \nu_{k}\right]$ is of the same form as $\mathbb{E}\left[P_{k+1}^{1,1} \mid \mathbf{P}_{k}, \nu_{k}\right]$ when $\mathbf{P}_{k}$ is replaced by $\hat{\mathbf{P}}_{k}$, and the Bellman equation for problem (27) is given by a similar Bellman equation to (20). The details are omitted for brevity.

\section{Structural Results on the Optimal TRANSMisSion POLICIES FOR SCALAR SYSTEMS}

This section presents structural results of the optimal transmission policies for scalar systems (where we will set $A=a$, $\left.C=1, \Sigma_{w}=\sigma_{w}^{2}, \Sigma_{v}=\sigma_{v}^{2}, \Sigma_{q}=\sigma_{q}^{2}\right)$ in the perfect packet receipt acknowledgments case examined in Section IV-A, which is also valid for the suboptimal solution presented in Section V. The idea is to apply the submodularity concept (see [39] and [40]) to the recursive Bellman (20), to show that the optimal policy $\nu^{o}(\cdot)$ in both scenarios is monotonically increasing with respect to the receiver's state estimation error variance $P^{1,1}$. This monotonicity then implies a threshold structure since the control space has only two elements $\{0,1\}$.

Definition 6.1 ([39] After [40]): A function $F(x, y): X \times$ $Y \rightarrow S$ is submodular in $(x, y)$ if $F\left(x_{1}, y_{1}\right)+F\left(x_{2}, y_{2}\right) \leq$ $F\left(x_{1}, y_{2}\right)+F\left(x_{2}, y_{1}\right)$ for all $x_{1}, x_{2} \in X$ and $y_{1}, y_{2} \in Y$ such that $x_{1} \geq x_{2}$ and $y_{1} \geq y_{2}$.

It is important to note that the submodularity is a sufficient condition for the optimality of monotone increasing policies. Specifically, if $F(x, y)$ defined above is submodular in $(x, y)$, then $y(x)=\arg \min _{y} F(x, y)$ is non-decreasing in $x$.
We define the ordering $\geq$ for matrices in class $\mathbb{S}$ of the form (25) as $\mathbf{P}_{1} \geq \mathbf{P}_{2}$ if $\mathbf{P}_{1}-\mathbf{P}_{2}$ is positive semidefinite. It is evident that for $\mathbf{P}_{1}, \mathbf{P}_{2} \in \mathbb{S}$, we have $\mathbf{P}_{1} \geq \mathbf{P}_{2}$ if and only if $P_{1}^{1,1} \geq P_{2}^{1,1}$. We also define $F: \mathbb{S} \times\{0,1\} \rightarrow \mathbb{S}$ as

$$
\begin{aligned}
F(\mathbf{P}, \nu)=\mathcal{A} \mathbf{P} \mathcal{A}^{T} & +Q-(1-p) \times\left[\mathcal{A} \mathbf{P} \mathcal{C}^{T}(\nu)+S\right] \\
& \times\left[\mathcal{C}(\nu) \mathbf{P} \mathcal{C}^{T}(\nu)+R\right]^{-1}\left[\mathcal{A} \mathbf{P} \mathcal{C}^{T}(\nu)+S\right]^{T}
\end{aligned}
$$

based on the instantaneous cost $\mathbb{E}[\mathcal{L}(\mathbf{P}, \gamma, \nu) \mid \mathbf{P}, \nu]$ in (22). Note that in the scalar case, $R$ can be made independent of $\nu_{k}$.

Lemma 6.1: The function $F(\mathbf{P}, \nu)$ is submodular in $(\mathbf{P}, \nu)$, that is, for $\mathbf{P}_{1}, \mathbf{P}_{2} \in \mathbb{S}$ such that $\mathbf{P}_{1} \geq \mathbf{P}_{2}$, we have

$$
F^{1,1}\left(\mathbf{P}_{1}, 1\right)+F^{1,1}\left(\mathbf{P}_{2}, 0\right) \leq F^{1,1}\left(\mathbf{P}_{1}, 0\right)+F^{1,1}\left(\mathbf{P}_{2}, 1\right)
$$

where $F^{1,1}(\cdot, \cdot)$ is the $(1,1)$ entry of $F(\cdot, \cdot)$. This implies that $F\left(\mathbf{P}_{1}, 1\right)+F\left(\mathbf{P}_{2}, 0\right) \leq F\left(\mathbf{P}_{1}, 0\right)+F\left(\mathbf{P}_{2}, 1\right)$.

\section{Proof: See Appendix C.}

We now present the relative value iteration algorithm to solve the Bellman (20). It is used to construct structural results for the optimal transmission policy. First, we consider the Bellman equation for the finite $T$-horizon stochastic control problem

$$
\begin{aligned}
V_{t}(\mathbf{P}) & =\min _{\nu \in\{0,1\}}\left(\mathbb{E}\left[\lambda \mathcal{L}^{1,1}(\mathbf{P}, \gamma, \nu)+(1-\lambda) J(\nu) \mid \mathbf{P}, \nu\right]\right. \\
& \left.+\mathbb{E}\left[V_{t+1}(\mathcal{L}(\mathbf{P}, \gamma, \nu)) \mid \mathbf{P}, \nu\right]\right), \quad 0 \leq t \leq T-1
\end{aligned}
$$

with terminal condition $V_{T}(\mathbf{P})=0$, where $T$ is large. We now define the function

$$
H_{t}(\cdot):=V_{t}(\cdot)-V_{t}\left(\mathbf{P}_{f}\right), \quad 0 \leq t \leq T
$$

where $\mathbf{P}_{f} \neq \mathbf{P}_{0}$ is fixed. We then have the following relative value iteration algorithm recursion:

$$
\begin{aligned}
H_{t}(\mathbf{P})=\min _{\nu \in\{0,1\}}\left(\mathbb{E}\left[\lambda \mathcal{L}^{1,1}(\mathbf{P}, \gamma, \nu)+(1-\lambda) J(\nu) \mid \mathbf{P}, \nu\right]\right. \\
\left.+\mathbb{E}\left[V_{t+1}(\mathcal{L}(\mathbf{P}, \gamma, \nu)) \mid \mathbf{P}, \nu\right]\right) \\
-\min _{\nu \in\{0,1\}}\left(\mathbb{E}\left[\lambda \mathcal{L}^{1,1}(\mathbf{P}, \gamma, \nu)+(1-\lambda) J(\nu) \mid \mathbf{P}=\mathbf{P}_{0}, \nu\right]\right. \\
\left.+\mathbb{E}\left[V_{t+1}(\mathcal{L}(\mathbf{P}, \gamma, \nu)) \mid \mathbf{P}=\mathbf{P}_{f}, \nu\right]\right)
\end{aligned}
$$

for $0 \leq t \leq T-1$. It can be shown that the relative value recursion (31) converges to the optimal solution $\rho$ of the infinite-time horizon average cost Bellman (20) such that $\rho \approx H_{0}\left(\mathbf{P}_{0}\right)$ (see the discussion in [36, p. 391]).

Theorem 6.1: The optimal transmission policy in the case of the perfect feedback channel is threshold with respect to the receiver's state estimation error variance $P^{1,1}$ (and, hence, in the augmented state estimation error covariance $\mathbf{P}$ ), i.e.,

$$
\nu^{o}(\mathbf{P})= \begin{cases}0, & \text { if } P_{k}^{1,1} \leq \phi^{*} \\ 1, & \text { otherwise }\end{cases}
$$

where $\phi^{*}$ is the threshold. 
Proof: See Appendix D.

The threshold structure of Theorem (6.1) simplifies the implementation of the optimal transmission policy significantly. However, this requires knowledge of the threshold $\phi^{*}(\cdot)$. In general, there is no closed-form expression for $\phi^{*}(\cdot)$, but it can be found via iterative search algorithms. Here, we present a stochastic gradient algorithm based on [41, Algorithm 1].

First, we establish some notation. For fixed $\mathbf{P}$, denote

$$
\begin{aligned}
J\left(\theta^{*}\right):=\mathbb{E}\left[\lambda \mathcal{L}^{1,1}\left(\mathbf{P}, \gamma, \nu^{o}\right)+\right. & \left.(1-\lambda) J(\nu) \mid \mathbf{P}, \nu^{o}\right] \\
& +\mathbb{E}\left[V_{0}\left(\mathcal{L}\left(\mathbf{P}, \gamma, \nu^{o}\right)\right) \mid \mathbf{P}, \nu^{o}\right]
\end{aligned}
$$

where the policy $\nu^{\circ}$ is defined in (32) based on the threshold $\phi^{*}$, and $V_{0}(\cdot)$ is obtained from the finite $T$-horizon Bellman (29). For $n \in \mathbb{N}, 0.5<\kappa \leq 1$ and $\omega, \varsigma>0$, we denote $\omega_{n}:=$ $\left(\omega /(n+1)^{\kappa}\right)$ and $\varsigma_{n}:=\left(\varsigma /(n+1)^{\kappa}\right)$.

Stochastic gradient algorithm for computing the threshold. For fixed $\mathbf{P}$ in the relative value algorithm (31), the following steps are carried out:

Step 1) Choose the initial threshold $\phi^{(0)}$.

Step 2) For iterations $n=0,1, \cdots$

- compute the gradient

$$
\partial_{\phi} J_{n}:=\frac{J\left(\phi^{(n)}+\omega_{n} d_{n}\right)-J\left(\phi^{(n)}-\omega_{n} d_{n}\right)}{2 \omega_{n}} d_{n}
$$

where $d_{n} \in\{-1,1\}$ is a random variable such that $\mathbb{P}\left(d_{n}=-1\right)=\mathbb{P}\left(d_{n}=1\right)=0.5$;

- update the threshold via $\phi^{(n+1)}=\phi^{(n)}-$ $\varsigma_{n} \partial_{\phi} J_{n}$ which gives

$$
\nu^{(n+1)}(\mathbf{P})= \begin{cases}0, & \text { if } P^{1,1} \leq \phi^{(n+1)} \\ 1, & \text { otherwise }\end{cases}
$$

The above algorithm is a gradient-estimate-based algorithm (see [42]) for estimating the optimal threshold $\phi^{*}(\cdot)$ where only measurements of the loss function are available (i.e., no gradient information). We note that (33) evaluates an approximation to the gradient. This algorithm generates a sequence of estimates for the threshold policy $\phi^{*}$ which converges to a local minimum with corresponding decision $\nu^{*}$. The reader is referred to [42] for associated convergence analysis of this and other related algorithms (see, for example, [42, Th. 7.1]). Note that gradient-estimate-based algorithms are sensitive to initial conditions and should be evaluated for several distinct initial conditions to find the best local minimum.

\section{NUMERICAL EXAMPLES}

Here, we present numerical results for a scalar model with parameters $a=0.95, c=1, \sigma_{w}^{2}=0.25, \sigma_{v}^{2}=0.01$, and $P_{x_{0}}=$ 1 in (1) and (2). These values give $P_{s}=0.26, K_{s}=0.91$, $K_{f}=0.96$, and $\Sigma_{s}=2.30$ in Section II-B. We take $\sigma_{q}^{2}=$ $\Sigma_{q}^{x}=\Sigma_{q}^{\epsilon}=0.01$ in (9) together with an optimal Lloyd-Max quantizer which yields $n_{0}=3$ and $n_{1}=5$ by (10). In the simulation results, an AWGN channel with BPSK modulation is assumed where $N_{0}=0.01$ in (12) (see Section II-D).

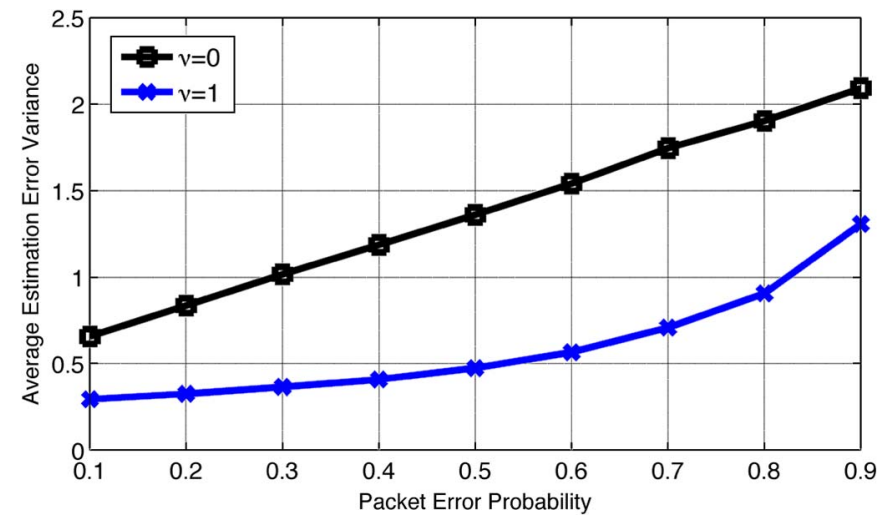

Fig. 2. Perfect feedback case: Average estimation error variance versus the packet error probabilities for the two cases of $\nu=0$ and $\nu=1$.

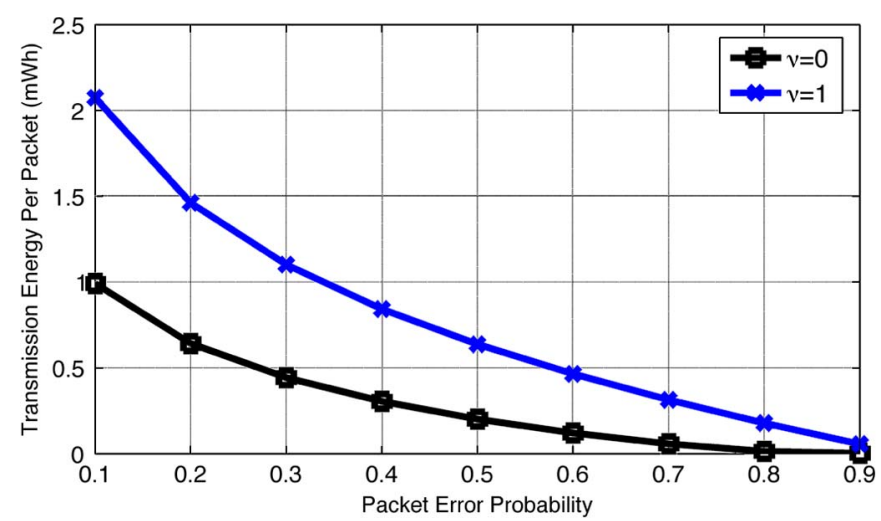

Fig. 3. Perfect feedback case: Transmission energy per packet (mWh) versus the packet error probabilities for the two cases of $\nu=0$ and $\nu=1$.

\section{A. Perfect Feedback Communication Channel Case}

First, let the packet error probability $p$ in (11) be equal to 0.2 . This gives $p_{b}^{0}=0.07$ and $p_{b}^{1}=0.04$ and, hence, energy per bit levels of $E_{b}^{0}=0.21$ and $E_{b}^{1}=0.29$, see Section IV.

In Fig. 2, we plot the average estimation error variance versus the packet error probabilities. More precisely, we take $\lambda=1$ in (18) without computing the optimal solution. Instead, we let the transmission policies $\left\{\nu_{k}, k \geq 0\right\}$ be fixed either equal to zero (sending innovations) or one (sending state estimates). On the other hand, Fig. 3 presents the packet transmission energy $J(\nu)$ [in milliwatt hours (mWh)] defined in Section IV versus the packet error probabilities. We let the transmission policy $\nu$ be fixed equal to either zero (sending innovations) or one (sending state estimates). Figs. 2 and 3 show that transmitting local estimates gives smaller error covariance, but also requires more transmit energy than transmitting local innovations to maintain the packet error probability. This motivates the optimization formulation (17).

We now set the weight $\lambda$ in (18) to 0.6 . The discretized equation of the relative value algorithm (31) is used for the numerical computation of the optimal transmission policy. In solving the Bellman (20), we use 40 discretization points for the state estimate error variance $P_{k}^{1,1}$ in the range of [0,2]. In Fig. 4, we plot the convex combination of the receiver's expected estimation error variance and the energy needed to transmit the packets, versus the packet loss probability $p \in$ 


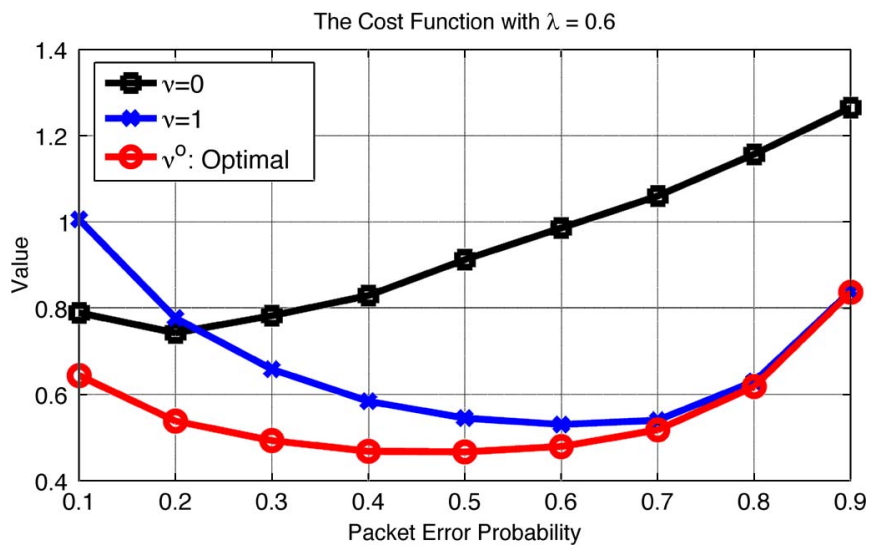

Fig. 4. Perfect feedback case: Performance versus the packet error probabilities.
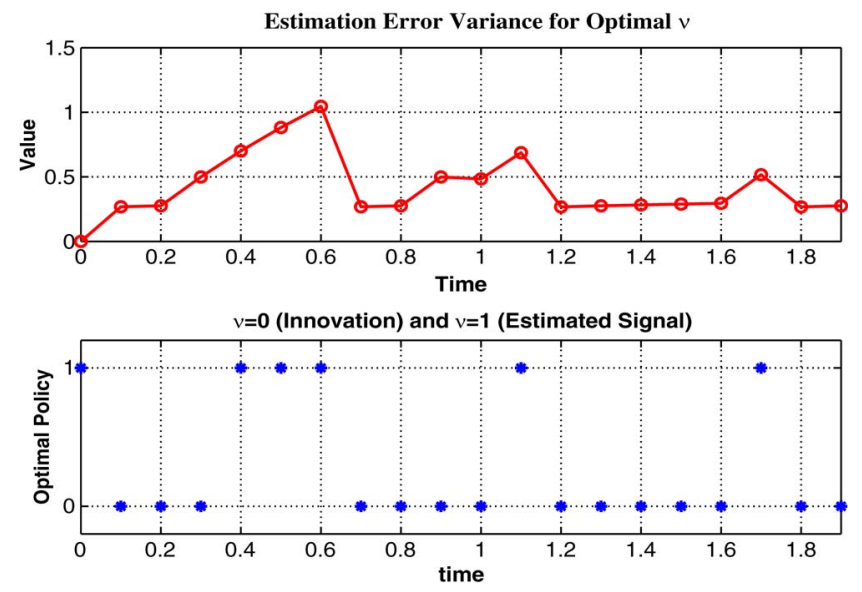

Fig. 5. Perfect feedback case: A single simulation run.

$[0.1,0.9]$ for the cases of: i) fixed transmission policy $\nu=0$, ii) fixed transmission policy $\nu=1$, and iii) optimal transmission policy $\nu^{o}$. We observe that for small packet loss probabilities, sending innovations $(\nu=0)$ is better than sending the state estimates $(\nu=1)$. On the other hand, for large packet loss probabilities, sending the state estimates gives better performance than sending the innovations, due to the poor estimation performance when sending innovations when the packet loss probability is high. Fig. 4 clearly shows that, especially at lowto-moderate packet error probabilities, the proposed method has the potential to give significant performance gains when compared to these fixed schemes.

Threshold Policy: With $p=0.2$ in (11), applying the stochastic gradient algorithm given at the end of Section VI with parameters $\omega=0.3, \varsigma=0.5$ and $\kappa=1$ yields the threshold $\phi^{*}=0.5$. For this case, a single run simulation result of the receiver's state estimation error variance $P_{(.)}^{1,1}$ is illustrated together with the optimal transmission strategy in Fig. 5.

\section{B. Imperfect Feedback Communication Channel Case}

We now consider the case of imperfect packet receipt acknowledgments, as described in Section II-E with parameters $\eta=0.4$ and $\delta=0.1$. Let $\lambda$ in (17) be equal to 0.6. The performance of the optimal and suboptimal solutions (of Section V)

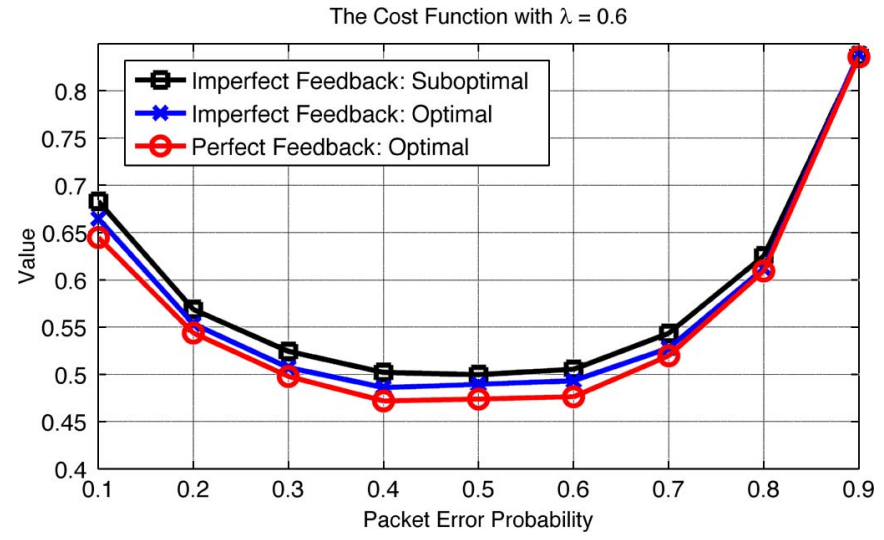

Fig. 6. Performance versus the packet error probabilities for optimal and suboptimal solutions for the imperfect feedback case, together with the performance of the optimal sequence in the perfect feedback case.

versus the packet loss probability $\mathbb{P}\left(\gamma_{k}=0\right)=p \in[0.1,0.9]$ is given in Fig. 6. The performance of the optimal sequence in the case of perfect packet receipt acknowledgments is also shown. We observe that for large packet error probabilities, the performance for the suboptimal solution, which is easier to implement, is close to the performance of the optimal solution.

\section{CONCLUSiOnS AND EXTENSIONS}

This paper presents a design methodology for remote estimation of the state of a stable linear stochastic dynamical system, subject to packet dropouts and unreliable acknowledgments. The key novelty of this formulation is that the smart sensor decides, at each discrete time instant, whether to transmit either its local state estimate or its local innovation. It is shown how to design optimal transmission policies in order to minimize a long-term average (infinite-time horizon) cost function as a convex combination of the receiver's expected estimation error variance and the energy needed to transmit the packets. Various computationally efficient suboptimal schemes are presented. For scalar systems, the optimality of a simple threshold policy in the case of perfect packet receipt acknowledgments is also proved.

The analysis of this paper can be extended to the case of unstable systems with some nontrivial modifications. In order to study unstable systems without feedback control, one can use the dynamic zoom-in zoom-out quantizer high-rate quantizers as used in [27] for decentralized Kalman filtering over bandwidth-constrained channels. In case of an unstable system stabilized via feedback control, the approach will likely be different and will possibly use the techniques of linear control design under signal-to-quantization-noise ratio constraints as investigated in [43] and [44]. These and other extensions will be investigated in a future work.

\section{APPENDIX}

\section{A. Proof of Theorem 3.1}

We have, by definition, $\mathcal{Y}_{k}^{s}=\sigma\left\{y_{t}: 0 \leq t \leq k\right\}$ and $\mathcal{Y}_{k}^{r}=$ $\sigma\left\{\gamma_{t}, \gamma_{t} \nu_{t}, \gamma_{t} z_{t}: 0 \leq t \leq k\right\}$. In addition, let us define the 
$\sigma$-fields: $\mathcal{Y}_{k}^{1}=\sigma\left\{\nu_{t}, y_{t}: 0 \leq t \leq k\right\}, \mathcal{Y}_{k}^{2}=\sigma\left\{\nu_{t}, z_{t}: 0 \leq t \leq\right.$ $k\}, \mathcal{Y}_{k}^{3}=\sigma\left\{\gamma_{t}, \nu_{t}, z_{t}: 0 \leq t \leq k\right\}$. We have the obvious inclusions $\mathcal{Y}_{k}^{2} \subseteq \mathcal{Y}_{k}^{1}$ and $\mathcal{Y}_{k}^{r} \subseteq \mathcal{Y}_{k}^{3}$. Now $\mathbb{E}\left[x_{k} \mid \mathcal{Y}_{k-1}^{s}\right]=\mathbb{E}\left[x_{k} \mid \mathcal{Y}_{k-1}^{1}\right]$ since $\nu_{k-1}$ does not provide any additional information about $x_{k}$ (because $\nu_{k}$ can depend on the error covariance but not the state). Then, we have

$$
\begin{aligned}
\mathbb{E}\left[\mathbb{E}\left[x_{k} \mid \mathcal{Y}_{k-1}^{s}\right] \mid \mathcal{Y}_{k-1}^{2}\right] & =\mathbb{E}\left[\mathbb{E}\left[x_{k} \mid \mathcal{Y}_{k-1}^{1}\right] \mid \mathcal{Y}_{k-1}^{2}\right] \\
& =\mathbb{E}\left[x_{k} \mid \mathcal{Y}_{k-1}^{2}\right]=\mathbb{E}\left[x_{k} \mid \mathcal{Y}_{k-1}^{3}\right]
\end{aligned}
$$

where the second equality is due to the inclusion $\mathcal{Y}_{k-1}^{2} \subseteq \mathcal{Y}_{k-1}^{1}$, and the third equality holds because $\gamma_{k-1}$ is independent of $x_{k}$. Following some additional manipulations and the fact that $\mathcal{Y}_{k-1}^{r} \subseteq \mathcal{Y}_{k-1}^{3}$, one can show that

$$
\mathbb{E}\left[\hat{x}_{k}^{s} \mid \mathcal{Y}_{k-1}^{r}\right]=\mathbb{E}\left[x_{k} \mid \mathcal{Y}_{k-1}^{r}\right]=\hat{x}_{k}^{r} .
$$

On the other hand

$$
\begin{aligned}
P_{k}^{2,2} \equiv & \mathbb{E}\left[\left(\hat{x}_{k}^{s}-\mathbb{E}\left[\hat{x}_{k}^{s} \mid \mathcal{Y}_{k-1}^{r}\right]\right)\left(\hat{x}_{k}^{s}-\mathbb{E}\left[\hat{x}_{k}^{s} \mid \mathcal{Y}_{k-1}^{r}\right]\right)^{T} \mid \mathcal{Y}_{k-1}^{r}\right] \\
=\mathbb{E} & {\left[\left(\hat{x}_{k}^{s}-\hat{x}_{k}^{r}\right)\left(\hat{x}_{k}^{s}-\hat{x}_{k}^{r}\right)^{T} \mid \mathcal{Y}_{k-1}^{r}\right] } \\
=\mathbb{E} & {\left[\left(\left(x_{k}-\hat{x}_{k}^{r}\right)-\left(x_{k}-\hat{x}_{k}^{s}\right)\right)\right.} \\
& \left.\quad \times\left(\left(x_{k}-\hat{x}_{k}^{r}\right)-\left(x_{k}-\hat{x}_{k}^{s}\right)\right)^{T} \mid \mathcal{Y}_{k-1}^{r}\right] \\
= & P_{k}^{1,1}+P_{s}-2 \mathbb{E}\left[\left(x_{k}-\hat{x}_{k}^{r}\right)\left(x_{k}-\hat{x}_{k}^{s}\right)^{T} \mid \mathcal{Y}_{k-1}^{r}\right]
\end{aligned}
$$

We note that $\tilde{x}_{k}^{s}:=x_{k}-\hat{x}_{k}^{s}$ is orthogonal to $\mathcal{Y}_{k-1}^{s}$ and, hence, orthogonal to $\mathcal{Y}_{k-1}^{r}$. Therefore, $\mathbb{E}\left[\hat{x}_{k}^{s}\left(\tilde{x}_{k}^{s}\right)^{T} \mid \mathcal{Y}_{k-1}^{r}\right]=0$ and $\mathbb{E}\left[\hat{x}_{k}^{r}\left(\tilde{x}_{k}^{s}\right)^{T} \mid \mathcal{Y}_{k-1}^{r}\right]=0$, which give

$$
\begin{aligned}
& \mathbb{E}\left[\left(x_{k}-\hat{x}_{k}^{r}\right)\left(x_{k}-\hat{x}_{k}^{s}\right)^{T} \mid \mathcal{Y}_{k-1}^{r}\right] \\
& \quad=\mathbb{E}\left[\left(\left(x_{k}-\hat{x}_{k}^{s}\right)+\left(\hat{x}_{k}^{s}-\hat{x}_{k}^{r}\right)\right)\left(x_{k}-\hat{x}_{k}^{s}\right)^{T} \mid \mathcal{Y}_{k-1}^{r}\right]=P_{s} .
\end{aligned}
$$

where the last equality holds because $\mathbf{P}_{k+1}$ is a function of $\mathbf{P}_{k}$, $\gamma_{k}$ and $\nu_{k}$, see (15). However, the chain rule implies that

$$
\begin{aligned}
\mathbb{P} & \left(\mathbf{P}_{k}, \gamma_{k}, z^{k}, \nu_{k}\right) \\
= & \mathbb{P}\left(\mathbf{P}_{k}, \gamma_{k}, z^{k-1}, \hat{\gamma}_{k}, \nu_{k-1}, \nu_{k}\right) \\
= & \mathbb{P}\left(\hat{\gamma}_{k} \mid \mathbf{P}_{k}, \gamma_{k}, z^{k-1}, \nu_{k-1}, \nu_{k}\right) \mathbb{P}\left(\gamma_{k} \mid \mathbf{P}_{k}, z^{k-1}, \nu_{k-1}, \nu_{k}\right) \\
& \times \mathbb{P}\left(\mathbf{P}_{k} \mid z^{k-1}, \nu_{k-1}, \nu_{k}\right) \mathbb{P}\left(z^{k-1}, \nu_{k-1}, \nu_{k}\right) \\
= & \mathbb{P}\left(\hat{\gamma}_{k} \mid \gamma_{k}\right) \mathbb{P}\left(\gamma_{k}\right) \mathbb{P}\left(\mathbf{P}_{k} \mid z^{k-1}, \nu_{k-1}\right) \mathbb{P}\left(z^{k-1}, \nu_{k-1}, \nu_{k}\right)
\end{aligned}
$$

Substituting (37) in (36) yields

$$
\begin{array}{r}
\mathbb{P}\left(\mathbf{P}_{k+1}, z^{k}, \nu_{k}\right)=\sum_{\gamma_{k}} \int_{P_{k}}\left(\mathbb{P}\left(\mathbf{P}_{k+1} \mid \mathbf{P}_{k}, \gamma_{k}, \nu_{k}\right) \mathbb{P}\left(\hat{\gamma}_{k} \mid \gamma_{k}\right)\right. \\
\left.\quad \times \mathbb{P}\left(\gamma_{k}\right) \mathbb{P}\left(\mathbf{P}_{k} \mid z^{k-1}, \nu_{k-1}\right) \mathbb{P}\left(z^{k-1}, \nu_{k-1}, \nu_{k}\right)\right) d P_{k} .
\end{array}
$$

On the other hand

$$
\mathbb{P}\left(\mathbf{P}_{k+1} \mid z^{k}, \nu_{k}\right)=\alpha \times \mathbb{P}\left(\mathbf{P}_{k+1}, z^{k} \nu_{k}\right)
$$

where $\alpha$ is a normalizing constant. Integrating (39) with respect to $\mathbf{P}_{k+1}$ gives $\alpha=\left(\int_{\mathbf{P}_{k+1}} \mathbb{P}\left(\mathbf{P}_{k+1}, z^{k}, \nu_{k}\right) d \mathbf{P}_{k+1}\right)^{-1}$. However

$$
\int_{\mathbf{P}_{k+1}} \mathbb{P}\left(\mathbf{P}_{k+1}, z^{k}, \nu_{k}\right) d \mathbf{P}_{k+1}
$$

$$
\begin{aligned}
=\int_{\mathbf{P}_{k+1}}[ & \sum_{\gamma_{k}} \int_{\mathbf{P}_{k}}\left(\mathbb{P}\left(\mathbf{P}_{k+1} \mid \mathbf{P}_{k}, \gamma_{k}, \nu_{k}\right) \mathbb{P}\left(\hat{\gamma}_{k} \mid \gamma_{k}\right) \mathbb{P}\left(\gamma_{k}\right)\right. \\
& \left.\left.\times \mathbb{P}\left(\mathbf{P}_{k} \mid z^{k-1}, \nu_{k-1}\right) \mathbb{P}\left(z^{k-1}, \nu_{k-1}, \nu_{k}\right)\right) d \mathbf{P}_{k}\right] d \mathbf{P}_{k+1} .
\end{aligned}
$$

This, together with (35), implies that $P_{k}^{2,2}=P_{k}^{1,1}-P_{s}$. In a similar way, it can be shown that $P_{k}^{1,2}=P_{k}^{1,1}-P_{s}$.

\section{B. Proof of Lemma 4.1}

The total probability formula ${ }^{4}$ and the chain rule give

$$
\begin{aligned}
& \mathbb{P}\left(\mathbf{P}_{k+1}, z^{k}, \nu_{k}\right) \\
& \quad=\sum_{\gamma_{k}} \int_{\mathbf{P}_{k}} \mathbb{P}\left(\mathbf{P}_{k+1}, \mathbf{P}_{k}, \gamma_{k}, z^{k}, \nu_{k}\right) d \mathbf{P}_{k} \\
& \quad=\sum_{\gamma_{k}} \int_{\mathbf{P}_{k}} \mathbb{P}\left(\mathbf{P}_{k+1} \mid \mathbf{P}_{k}, \gamma_{k}, z^{k}, \nu_{k}\right) \mathbb{P}\left(\mathbf{P}_{k}, \gamma_{k}, z^{k}, \nu_{k}\right) d \mathbf{P}_{k} \\
& \quad=\sum_{\gamma_{k}} \int_{\mathbf{P}_{k}} \mathbb{P}\left(\mathbf{P}_{k+1} \mid \mathbf{P}_{k}, \gamma_{k}, \nu_{k}\right) \mathbb{P}\left(\mathbf{P}_{k}, \gamma_{k}, z^{k}, \nu_{k}\right) d \mathbf{P}_{k}
\end{aligned}
$$

${ }^{4} \mathbb{P}(A, \mid, B)=\sum_{i} \mathbb{P}\left(A, C_{i}, \mid, B\right)$
With some additional calculations, one can show that

$$
\alpha=\left(\mathbb{P}\left(z^{k-1}, \nu_{k-1}, \nu_{k}\right) \sum_{\gamma_{k}} \mathbb{P}\left(\hat{\gamma}_{k} \mid \gamma_{k}\right) \mathbb{P}\left(\gamma_{k}\right)\right)^{-1} .
$$

Finally, substituting (38) and (41) in (39) gives (24).

\section{Proof of Lemma 6.1}

Let $\mathbf{P}:=\left[\begin{array}{lll}P^{1,1} & P^{1,1}-P_{s} P^{1,1}-P_{s} P^{1,1}-P_{s}\end{array}\right]$ where $P^{1,1} \geq P_{s}$ which implies that $\mathbf{P}_{1} \in \mathbb{S}$. First, note that

$$
F^{1,1}(\mathbf{P}, 0)=a^{2} P^{1,1}+\sigma_{w}^{2}-(1-p) \times \frac{a^{2} K_{f}^{2} P_{s}^{2}}{K_{f}^{2} P_{s}+R}
$$

and

$F^{1,1}(\mathbf{P}, 1)=a^{2} P^{1,1}+\sigma_{w}^{2}-(1-p) \times \frac{a^{2}\left(\left(P^{1,1}-P_{s}\right)+K_{f} P_{s}\right)^{2}}{\left(P^{1,1}-P_{s}\right)+K_{f}^{2} P_{s}+R}$. 
We denote $g(x):=\left(\left(\left(x-P_{s}\right)+K_{f} P_{s}\right)^{2} /\left(x-P_{s}\right)+K_{f}^{2} P_{s}+R\right)$ for $x \geq P_{s}$. Let $\mathbf{P}_{1}, \mathbf{P}_{2} \in \mathbb{S}$ be such that $\mathbf{P}_{1} \geq \mathbf{P}_{2}$, then the inequality (28) is equivalent to

$$
\begin{array}{r}
a^{2}\left(P_{1}^{1,1}-P_{2}^{1,1}\right)-(1-p) a^{2}\left(g\left(P_{1}^{1,1}\right)-g\left(P_{2}^{1,1}\right)\right) \\
\leq a^{2}\left(P_{1}^{1,1}-P_{2}^{1,1}\right) .
\end{array}
$$

Again, after some additional algebra, one can show that $g^{\prime}(x) \geq 0$ for $x \geq P_{s}$. This, together with $P_{1}^{1,1} \geq P_{2}^{1,1} \geq P_{s}$, implies that the inequality (42) is valid. This gives (28); thus, $F\left(\mathbf{P}_{1}, 1\right)-F\left(\mathbf{P}_{2}, 1\right) \leq F\left(\mathbf{P}_{1}, 0\right)-F\left(\mathbf{P}_{2}, 0\right)$ based on Theorem 3.1 and the fact that for $\mathbf{P}_{1}, \mathbf{P}_{2} \in \mathbb{S}$, we have $\mathbf{P}_{1} \geq$ $\mathbf{P}_{2}$ if and only if $P_{1}^{1,1} \geq P_{2}^{1,1}$.

\section{Proof of Theorem 6.1}

Based on the relative value iteration (31), define

$$
\begin{aligned}
L_{t}(\mathbf{P}, \nu)= & \mathbb{E}\left[\lambda \mathcal{L}^{1,1}(\mathbf{P}, \gamma, \nu)+(1-\lambda) J(\nu) \mid \mathbf{P}, \nu\right] \\
& +\mathbb{E}\left[V_{t+1}(\mathcal{L}(\mathbf{P}, \gamma, \nu)) \mid \mathbf{P}, \nu\right] \\
= & : L_{t}^{(1)}(\mathbf{P}, \nu)+L_{t}^{(2)}(\mathbf{P}, \nu)
\end{aligned}
$$

for $0 \leq t \leq T-1$

Submodularity of $L_{t}^{(1)}(\mathbf{P}, \nu)$ : Lemma 6.1 implies that $F(\mathbf{P}, \nu)=\mathbb{E}[\mathcal{L}(\mathbf{P}, \gamma, \nu) \mid \mathbf{P}, \nu]$ and, hence, $(F(P, \nu))^{1,1}=$ $\mathbb{E}\left[\mathcal{L}^{1,1}(\mathbf{P}, \gamma, \nu) \mid \mathbf{P}, \nu\right]$ are submodular in $(\mathbf{P}, \nu)$. It is evident that $\mathbb{E}[J(\nu) \mid \nu]$ is also submodular in $(\mathbf{P}, \nu)$ since it is independent of $\mathbf{P}$. Therefore, their convex combination $L_{t}^{(1)}(\mathbf{P}, \nu)$ is submodular in $(\mathbf{P}, \nu)$.

Submodularity of $L_{t}^{(2)}(\mathbf{P}, \nu)$ : First, we note that $\mathcal{L}(\mathbf{P}, \gamma, 0)=$ $\mathbb{E}[\mathcal{L}(\mathbf{P}, \gamma, \nu) \mid \mathbf{P}, \nu=0]$ and $\mathcal{L}(\mathbf{P}, \gamma, 1)=\mathbb{E}[\mathcal{L}(\mathbf{P}, \gamma, \nu) \mid \mathbf{P}, \nu=1]$ given in (22) are concave ${ }^{5}$ and nondecreasing functions in $\mathbf{P}$ (see [45, Lemmas 1 and 2]). This implies that $\mathbb{E}\left[\lambda \mathcal{L}^{1,1}(\mathbf{P}, \gamma, \nu)+\right.$ $(1-\lambda) J(\nu) \mid \mathbf{P}, \nu=0], \quad \mathbb{E}\left[\lambda \mathcal{L}^{1,1}(\mathbf{P}, \gamma, \nu)+(1-\lambda) J(\nu) \mid \mathbf{P}\right.$, $\nu=1]$ and therefore

$$
\min _{\nu \in\{0,1\}}\left(\mathbb{E}\left[\lambda \mathcal{L}^{1,1}(\mathbf{P}, \gamma, \nu)+(1-\lambda) J(\nu) \mid \mathbf{P}, \nu\right]\right)
$$

are concave and nondecreasing functions of $\mathbf{P}$ (note that the expectation operator preserves concavity). By induction and the fact that the composition of two nondecreasing concave functions is itself concave and nondecreasing, one can show that the value function $V_{t}(\mathbf{P})$ in (29) is a concave and nondecreasing function of $\mathbf{P}$. The composition of a nondecreasing concave function $V_{t}(\cdot)$ with a monotonic submodular function $\mathcal{L}(\cdot, \gamma, \nu)$ is submodular (see [46, Prop. 2.3.5, part (c)]). Therefore, $L_{t}^{(2)}(\mathbf{P}, \nu)=\mathbb{E}\left[V_{t+1}(\mathcal{L}(\mathbf{P}, \gamma, \nu)) \mid \mathbf{P}, \nu\right]$ is submodular in $(\mathbf{P}, \nu)$. Finally, the submodularity of $L_{t}(\mathbf{P}, \nu)$ follows from the fact that the sum of two submodular functions is also submodular.

Consequently, for $0 \leq t \leq T-1, \arg \min _{\nu \in\{0,1\}} L_{t}(\mathbf{P}, \nu)$ is nondecreasing in $\mathbf{P}$ and, hence, nondecreasing in $P^{1,1}$. This monotonicity implies the threshold structure (32) since the control space has only two elements $\{0,1\}$ (see [40]).

\footnotetext{
${ }^{5}$ The proof of concavity is based on the fact that a function $f(x)$ is concave in $x$ if and only if $f\left(x_{0}+t h\right)$ is concave in the scalar $t$ for all $x_{0}$ and $h$.
}

\section{REFERENCES}

[1] J. Baillieul and P. J. Antsaklis, "Control and communication challenges in networked real-time systems," Proc. IEEE, vol. 95, no. 1, pp. 9-28, Jan. 2007.

[2] G. N. Nair and R. J. Evans, "Stabilizability of stochastic linear systems with finite feedback data rates," SIAM J. Control Optim., vol. 43, no. 2, pp. 413-436, 2004.

[3] P. Minero, L. Coviello, and M. Franceschetti, "Stabilization over Markov feedback channels: The general case," IEEE Trans. Autom. Control, vol. 58, no. 2, pp. 349-362, Feb. 2013.

[4] G. N. Nair, F. Fagnani, S. Zampieri, and R. J. Evans, "Feedback control under data rate constraints: An overview," Proc. IEEE, vol. 95, no. 1, pp. 108-137, Jan. 2007.

[5] B. Sinopoli et al., "Kalman filtering with intermittent observations," IEEE Trans. Autom. Control, vol. 49, no. 9, pp. 1453-1464, Sep. 2004.

[6] Y. Xu and J. P. Hespanha, "Estimation under uncontrolled and controlled communications in networked control systems," in Proc. 44th IEEE CDC, Seville, Spain, Dec. 2005, pp. 842-847.

[7] M. Huang and S. Dey, "Stability of Kalman filtering with Markovian packet losses," Automatica, vol. 43, pp. 598-607, 2007.

[8] M. Epstein, L. Shi, A. Tiwari, and R. M. Murray, "Probabilistic performance of state estimation across a lossy network," Automatica, vol. 44, pp. 3046-3053, Dec. 2008.

[9] L. Schenato, "Optimal estimation in networked control systems subject to random delay and packet drop," IEEE Trans. Autom. Control, vol. 53, no. 5, pp. 1311-1317, Jun. 2008.

[10] Y. Mo and B. Sinopoli, "A characterization of the critical value for Kalman filtering with intermittent observations," in Proc. 47th IEEE CDC, Cancun, Mexico, 2008, pp. 2692-2697.

[11] D. E. Quevedo, A. Ahlén, A. S. Leong, and S. Dey, "On Kalman filtering over fading wireless channels with controlled transmission powers," Automatica, vol. 48, no. 7, pp. 1306-1316, July 2012.

[12] L. Schenato, B. Sinopoli, M. Franceschetti, K. Poolla, and S. S. Sastry, "Foundations of control and estimation over lossy networks," Proc. IEEE, vol. 95, no. 1, pp. 163-187, Jan. 2007.

[13] D. E. Quevedo, A. Ahlén, and J. Østergaard, "Energy efficient state estimation with wireless sensors through the use of predictive power control and coding," IEEE Trans. Signal Process., vol. 58, no. 9, pp. 4811-4823, Sep. 2010.

[14] A. S. Leong and S. Dey, "Power allocation for error covariance minimization in $\{\mathrm{K}\}$ alman filtering over packet dropping links," in Proc. 51 st IEEE CDC, Maui, HI, USA, Dec. 2012, pp. 3335-3340.

[15] M. Nourian, A. S. Leong, and S. Dey, "Optimal energy allocation for Kalman filtering over packet dropping links with imperfect acknowledgments and energy harvesting constraints," IEEE Trans. Autom. Control, vol. 59, no. 8, pp. 2128-2143, Aug. 2014.

[16] K. You and L. Xie, "Minimum data rate for mean square stabilization of discrete LTI systems over lossy channels," IEEE Trans. Autom. Control, vol. 55, no. 10, pp. 2373-2378, Oct. 2010.

[17] K. You and L. Xie, "Minimum data rate for mean square stabilizability of linear systems with Markovian packet losses," IEEE Trans. Autom. Control, vol. 56, no. 4, pp. 772-785, Apr. 2011.

[18] K. Okano and H. Ishii, "Stabilization of uncertain systems with finite data rates and Markovian packet losses," in Proc. Eur. Control Conf., 2013, pp. 2368-2373.

[19] M. Trivellato and N. Benvenuto, "State control in networked control systems under packet drops and limited transmission bandwidth," IEEE Trans. Commun., vol. 58, no. 2, pp. 611-622, Feb. 2010.

[20] D. E. Quevedo, J. Ø stergaard, and D. Nešić, "Packetized predictive control of stochastic systems over bit-rate limited channels with packet loss," IEEE Trans. Autom. Control, vol. 56, no. 12, pp. 2854-2868, Dec. 2011.

[21] S. Dey, A. Chiuso, and L. Schenato, "Remote estimation subject to packet loss and quantization noise," in Proc. 52nd IEEE CDC, Dec. 2013, pp. 6097-6104.

[22] B. D. O. Anderson and J. B. Moore, Optimal Filtering. Englewood Cliffs, NJ, USA: Prentice-Hall, 1979.

[23] D. E. Quevedo, A. Ahlén, J. Østergaard, and G. C. Goodwin, "Innovations-based state estimation with wireless sensor networks," in Proc. ECC, Budapest, Hungary, 2009, pp. 4858-4864.

[24] N. S. Jayant and P. Noll, Digital Coding of Waveforms. Englewood Cliffs, NJ, USA: Prentice-Hall, 1984.

[25] D. Marco and D. L. Neuhoff, "The validity of the additive noise model for uniform scalar quantizers," IEEE Trans. Inf. Theory, vol. 51, no. 5, pp. 1739-1755, May 2005.

[26] V. K. Goyal, "High-rate transform coding: How high is high, does it matter?" in Proc. ISIT, Sorrento, Italy, 2000, p. 207. 
[27] A. S. Leong, S. Dey, and G. N. Nair, "Quantized filtering schemes for multi-sensor linear state estimation: Stability and performance under high rate quantization," IEEE Trans. Signal. Process., vol. 61, no. 15, pp. 3852-3865, Aug. 2013.

[28] P. W. Moo, "Asymptotic Analysis of Lattice-Based Quantization," Ph.D. dissertation, Dept. of Electrical Eng. and Computer Science, University of Michigan, Ann Arbor, Michigan, USA, 1998.

[29] A. Gersho, "Asymptotically optimal block quantization," IEEE Trans. Inf. Theory, vol. IT-25, no. 4, pp. 373-380, Jul, 1979.

[30] R. M. Gray and D. L. Neuhoff, "Quantization," IEEE Trans. Inf. Theory, vol. 44, no. 6, pp. 2325-2383, Oct. 1998.

[31] R. Zamir and M. Feder, "On lattice quantization noise," IEEE Trans. Inf. Theory, vol. 42, no. 4, pp. 1152-1159, Jul. 1996.

[32] J. G. Proakis, Digital Communications, 4th ed. New York, USA: McGraw-Hill, 2001.

[33] E. Garone, B. Sinopoli, and A. Casavola, "LQG control over lossy TCP-like networks with probabilistic packet acknowledgements," Int. J. Syst. Control Commun., vol. 2, no. 1-3, pp. 55-81, 2010.

[34] M. Moayedi, Y. K. Foo, and Y. C. Soh, "Networked LQG control over unreliable channels," Int. J. Robust Nonlin. Control, vol. 23, no. 2, pp. 167189, 2013.

[35] O. C. Imer, S. Yüksel, and T. Başar, "Optimal control of LTI systems over unreliable communication links," Automatica, vol. 42, no. 9, pp. 14291439, Sep. 2006.

[36] D. P. Bertsekas, Dynamic Programming and Optimal Control, 2nd ed, vol. 1. Belmont, MA, USA: Athena Scientific, 1995.

[37] A. Arapostathis, V. S. Borkar, E. Fernáandez-Gaucherand, M. K. Ghosh, and S. I. Marcus, "Discrete-time controlled Markovprocesses with average cost criterion: A survey," SIAM J. Control Optim., vol. 31, no. 2 , pp. 282-344, 1993.

[38] P. R. Kumar and P. Varaiya, Stochastic Systems: Estimation, Identification and Adaptive Control. Englewood Cliffs, NJ, USA: Prentice-Hall, 1986.

[39] M. H. Ngo and V. Krishnamurthy, "Optimality of threshold policies for transmission scheduling in correlated fading channels," IEEE Trans. Commun., vol. 57, no. 8, pp. 2474-2483, Aug. 2009.

[40] D. M. Topkis, Supermodularity and Complementarity. Princeton, NJ, USA: Princeton Univ. Press, 2001.

[41] V. Krishnamurthy, R. Bitmead, M. Gevers, and E. Miehling, "Sequential detection with mutual information stopping cost," IEEE Trans. Signal Process., vol. 60, no. 2, pp. 700-714, Feb. 2012.

[42] J. C. Spall, Introduction to Stochastic Search and Optimization: Estimation, Simulation, and Control. New York, USA: Wiley, 2005.

[43] A. Chiuso, N. Laurenti, L. Schenato, and A. Zanella, "LQG cheap control over SNR-limited lossy channels with delay," in Proc. IEEE Conf. Dec. Control, Dec. 2013, pp. 3988-3993.

[44] E. I. Silva, G. C. Goodwin, and D. E. Quevedo, "Control system design subject to SNR constraints," Automatica, vol. 46, no. 2, pp. 428-436, Feb. 2010.

[45] V. Gupta, T. H. Chung, B. Hassibi, and R. M. Murray, "On a stochastic sensor selection algorithm with applications in sensor scheduling and sensor coverage," Automatica, vol. 42, no. 2, pp. 251-260, 2006.

[46] D. Simchi-Levi, X. Chen, and J. Bramel, The Logic of Logistics: Theory, Algorithms, Applications for Logistics and Supply Chain Management. Berlin, Germany: Springer-Verlag, 2004.

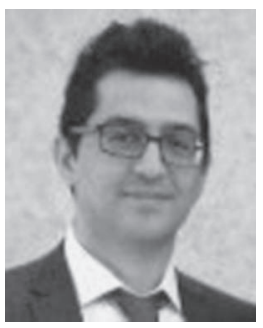

Mojtaba Nourian (S'06-M'12) received dual B.Sc. degrees in applied mathematics and in electrical engineering and the M.Sc. degree in applied mathematics from Amirkabir University of Technology (Tehran Polytechnic), Tehran, Iran, in 2005 and 2007, respectively, and the Ph.D. degree in electrical engineering from McGill University, Montreal, QC, Canada, in 2012.

Currently, he is a Research Fellow in the Department of Electrical and Electronic Engineering, University of Melbourne, Melbourne, Australia. His current research interests include mean field stochastic control, signal processing for sensor networks, and networked system estimation and control.

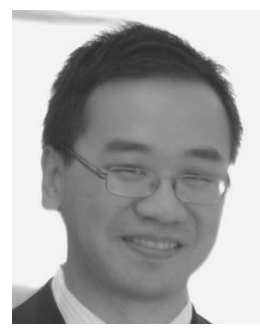

Alex S. Leong (M'08) was born in Macau in 1980. He received the B.S. degree in mathematics, the B.E. degree in electrical engineering, and the Ph.D. degree in electrical engineering from the University of Melbourne, Parkville, Australia, in 2003 and 2008.

Currently, he is a Research Fellow with the Department of Electrical and Electronic Engineering, University of Melbourne, Parkville, Australia. His research interests include statistical signal processing, signal processing for sensor networks, and networked control systems.

Dr. Leong was the recipient of the L. R. East Medal from Engineers Australia in 2003, and an Australian Postdoctoral Fellowship from the Australian Research Council in 2009.

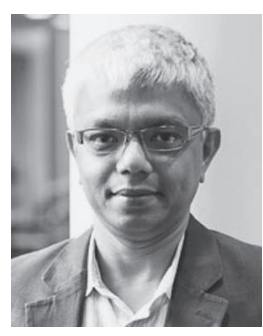

Subhrakanti Dey (M'96-SM'06) was born in India in 1968. He received the B.Tech. and M.Tech. degrees in electronics and electrical communication engineering from the Indian Institute of Technology, Kharagpur, India, in 1991 and 1993, respectively, and the Ph.D. degree in systems engineering from the Research School of Information Sciences and Engineering, Australian National University, Canberra, Australia, in 1996.

Currently, he is a Professor in the Department of Engineering Sciences at Uppsala University, Uppsala, Sweden. Previously, he was a Professor with the Department of Electrical and Electronic Engineering, University of Melbourne, Parkville, Australia, from 2000 to 2013. From 1995 to 1997 and from 1998 to 2000, he was a Postdoctoral Research Fellow with the Department of Systems Engineering, Australian National University. From 1997 to 1998, he was a Postdoctoral Research Associate with the Institute for Systems Research, University of Maryland, College Park, MD, USA. His current research interests include networked control systems, wireless communications and networks, signal processing for sensor networks, as well as stochastic and adaptive estimation and control.

Prof. Dey currently serves on the Editorial Board of IEEE TRANSACTIONS on Signal Processing and Systems and Control Letters. He was also an Associate Editor for the IEEE TRANSACTIONS ON SignAl PROCESSING during 2007-2010 and the IEEE TRANSACTIONS ON AUTOMATIC CONTROL during 2004-2007.

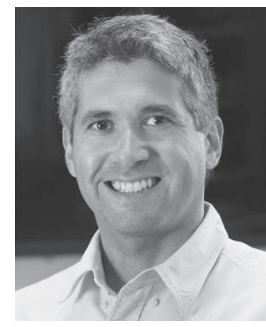

Daniel E. Quevedo (S'97-M'05-SM'14) received Ingeniero Civil Electrónico and Magister en Ingeniería Electrónica degrees from the Universidad Técnica Federico Santa María, Valparaíso, Chile, in 2000 , and the Ph.D. degree in the field of systems and control theory from The University of Newcastle, Newcastle, Australia, in 2005.

Currently, he is an Associate Professor with The University of Newcastle. He has been a Visiting Researcher at various institutions, including Uppsala University, Uppsala, Sweden; KTH Stockholm, Stockholm, Sweden; Aalborg University, Aalborg, Denmark; Kyoto University, Kyoto, Japan; INRIA Grenoble, France; University of Notre Dame, Notre Dame, IN, USA; and The Hong Kong University of Science and Technology, Hong Kong, China.

Dr. Quevedo was supported by a full scholarship from the alumni association during his time at the Universidad Técnica Federico Santa María and received several university-wide prizes upon graduating. He received the IEEE Conference on Decision and Control Best Student Paper Award in 2003 and was also a finalist in 2002. In 2009, he was awarded a five-year Australian Research Fellowship. His research interests include several areas within automatic control, signal processing, and power electronics. 